\title{
CLASSIFICATION OF SURFACES IN THREE-SPHERE IN LIE SPHERE GEOMETRY
}

\author{
TAKAYOSHI YAMAZAKI AND ATSUKO YAMADA YOSHIKAWA
}

\section{Introduction}

We studied plane curves in Lie sphere geometry in [YY]. Especially we constructed Lie frames of curves in $S^{2}$ and classified them by the Lie equivalence. In this paper we are concerned with surfaces in $S^{3}$. We construct Lie frames and classify them. We moreover obtain the necessary and sufficient condition that two surfaces are Lie equivalent.

We give some basic concepts about frames before explaining our main result. Let $N$ be a smooth $n$-dimensional manifold, and let $\lambda: M \rightarrow N$ and $\tilde{\lambda}: \tilde{M} \rightarrow N$ be embedded submanifolds of dimension $m$. We say that $\lambda$ and $\tilde{\lambda}$ have contact of at least order $k$ at $p \in M$ and $\tilde{p} \in \tilde{M}$ if $\lambda$ and $\tilde{\lambda}$ agree up to the differentials of order $k$ at $p$ and $\tilde{p}$. Let $G$ be a group of diffeomorphisms on $N$. We say that $\lambda$ and $\bar{\lambda}$ have $G$-contact of at least order $k$ at $p$ and $\tilde{p}$ if there exists a $P \in G$ such that $\lambda$ and $P \circ \tilde{\lambda}$ have contact of at least order $k$ at $p$ and $\tilde{p}$.

Let $\lambda: M \rightarrow G / H$ be a connected, smoothly embedded $n$-dimensional submanifold of a homogeneous space $G / H$. We state the definition of Frenet frames of $\lambda$ and its construction following G. R. Jensen [J]. Firstly we construct the set of zeroth order frames. A zeroth order frame at $p \in M$ is an element $P \in G$ such that $\pi(P)=\lambda(p)$ (where $\pi$ is the natural projection $G \rightarrow G / H$ ). Let $L_{0}$ denote the set of all zeroth order frames on $M$. A zeroth order frame field $u$ along $\lambda$ is a smooth cross section of $L_{0} \rightarrow M$.

Secondly we construct first order frames. We denote by $g$ and $\mathfrak{h}$ the Lie algebras of $G$ and $H$ respectively. We take a vector subspace $\mathfrak{m}$ of $\mathfrak{g}$ complementary to $\mathfrak{h}$, and choose a basis $e_{1}, \ldots, e_{m_{0}}$ of $\mathfrak{m}$. With respect to this basis, we consider the isotropy representation $\rho_{0}: H \rightarrow G L\left(m_{0}, \mathbf{R}\right)$ given by the adjoint action of $H$ on $\mathfrak{g} / \mathfrak{h} \cong \mathfrak{m}$. There is a naturally defined smooth map $\lambda_{0}$ from $L_{0}$ to the Grassmann manifold $G_{m_{0}, n}$ given by $\lambda_{0}(u)=u_{*}^{-1} \lambda_{*} M_{p}$ where $\lambda(p)=\pi(u)$. We choose a local

Received February 6, 1995. 
cross section $W_{1}$ of the action $\left(H, \rho_{0}\right)$ on $G_{m_{0}, n}$. If there exists a zeroth order frame field $u$ along $\lambda$ such that $\lambda_{0}(u) \subset W_{1}$, we let $L_{1}=\lambda_{0}^{-1}\left(W_{1}\right)$ and call $L_{1}$ the set of first order frames on $\lambda$ (with respect to $W_{1}$ ). We define a first order frame field along $\lambda$ to be a smooth cross section $L_{1} \rightarrow M$.

Furthermore we iterate this construction of frames; $L_{0} \supset L_{1} \supset L_{2} \supset \cdots \supset$ $L_{k} \supset \cdots$. Thus we construct a set of $k$-th order frames $L_{k}$ which gives $k$-th order contact under the action of $G$. Suppose frames of all orders can be constructed on $\lambda$. The sequence $\operatorname{dim} L_{0} \geq \operatorname{dim} L_{1} \geq \operatorname{dim} L_{2} \geq \cdots$ eventually stabilizes. Thus there is the smallest integer $q \geq 1$ such that $\operatorname{dim} L_{k}=\operatorname{dim} L_{q}$ for all $k \geq q$. Then the frames of order $q$ are called the Frenet frames of $\lambda$.

Let us now return to the gist of this paper. Let $S^{n}$ be the unit sphere in the Euclidean space $\mathbf{E}^{n+1}$, and $T_{1} S^{n}$ the unit tangent bundle of $S^{n}$, i.e. $T_{1} S^{n}=\{(u, v)$ $\left.\in S^{n} \times S^{n} ; u \cdot v=0\right\}$, where $\cdot$ denotes the inner product of $\mathbf{E}^{n+1}$. An immersed hypersurface $f: M^{n-1} \rightarrow S^{n}$ with a unit normal field $\xi: M^{n-1} \rightarrow S^{n}$ naturally induces a map $\lambda=(f, \xi): M^{n-1} \rightarrow T_{1} S^{n}$. This map $\lambda$ is called the Legendre map induced by $f$ with $\xi$.

Let $P O(n+1,2)$ be the projective orthogonal group of signature $(n+1,2)$. The group $P O(n+1,2)$ acts on $T_{1} S^{n}$ transitively. Then $T_{1} S^{n}$ is equal to a homogeneous space $P O(n+1,2) / H$ for the isotropy subgroup $H$ of $P O(n+1,2)$ at a point. Let $\lambda: M^{n-1} \rightarrow T_{1} S^{n}$ and $\tilde{\lambda}: \tilde{M}^{n-1} \rightarrow T_{1} S^{n}$ be embedded Legendre maps. We say that $\lambda$ and $\tilde{\lambda}$ are Lie equivalent if $\lambda$ and $\tilde{\lambda}$ are $P O(n+1,2)$ congruent, that is, there exists a $P \in P O(n+1,2)$ such that $P \lambda(M)$ agree with $\tilde{\lambda}(\tilde{M})$. Frenet frames of a Legendre map $\lambda$ in $T_{1} S^{n}$ under the action of $P O(n+1,2)$ are called Lie frames of $\lambda$. We are here concerned with the case when $n=3$.

We summarize our main results as follows: Let $f: M^{2} \rightarrow S^{3}$ be an embedded surface with a field of unit normals $\xi: M^{2} \rightarrow S^{3}$, and $\lambda=(f, \xi): M^{2} \rightarrow T_{1} S^{3}$ be the Legendre map induced by $f$ with $\xi$. Let $\Omega$ be the Maurer-Cartan form on $P O(4,2)$. Then for $\lambda$ we can construct a Lie frame $u: M^{2} \rightarrow P O(4,2)$ of one of the distinct five types (Type (a),..., Type (e) in Theorem 3.1) with respect to the pullback of $\Omega$ by $u$. Furthermore, a surface of Type (a) is an oriented sphere,

Type (b) is a cyclide of Dupin,

Type (c) or (d) is a "degenerate" surface (including canal surface) and Type (e) is a general surface.

In Section 1, we outline basic facts in Lie sphere geometry. This section is largely based on U. Pinkall [P] an T. E. Cecil [C]. In Section 2, we give some concepts about frames according to G. R. Jensen [J]. In Section 3, we give our main theorem and its proof. In Section 4, we get some characteristics of surfaces in Lie equivalent classes. U. Pinkall showed that the class of Dupin hypersurfaces in $S^{n}$ 
is invariant under Lie transformations $([\mathrm{P}])$. We see here that the degeneration of Lie frames characterizes oriented spheres, cyclides of Dupin and canal surfaces in $T_{1} S^{3}$.

We wish to thank Professor Hajime Sato for his helpful suggestions and comments in studying the problems here.

\section{Lie sphere geometry}

\subsection{Lie spheres and Lie transformations}

Let $S^{n}$ be the unit sphere in the Euclidean space $\mathbf{E}^{n+1}$, and $T_{1} S^{n}$ the unit tangent bundle of $S^{n}$; i.e.

$$
T_{1} S^{n}=\left\{(u, v) \in S^{n} \times S^{n} ; u \cdot v=0\right\},
$$

where $\cdot$ denotes the inner product of $\mathbf{E}^{n+1}$. A hypersphere $\iota: S^{n-1} \rightarrow S^{n}$ with a unit normal vector field $\xi$ along $\iota$ which gives an orientation induces a mapping $(\iota, \xi): S^{n-1} \rightarrow T_{1} S^{n}$. We call also $(\iota, \xi)$ an oriented hypersphere. When $\iota$ shrinks to a point, we regard $\xi$ as the inclusion of the fiber of $T_{1} S^{n}$ over the point $\iota$ into $T_{1} S^{n}$, and call $(\iota, \xi)$ a point sphere. We use the term Lie sphere to denote an oriented hypersphere or a point sphere.

Let $\mathbf{R}_{2}^{n+3}=\left\{x=\left(x_{1}, \cdots, x_{n+3}\right) ; x_{i} \in \mathbf{R}\right\}$ be an $(n+3)$-dimensional real vector space with the scalar product $\langle$, $\rangle$ defined by

$$
\langle x, y\rangle={ }^{t} x S y,
$$

where

$$
S=\left(S_{i}\right)=\left(\begin{array}{ccc}
0 & 0 & -I_{2} \\
0 & I_{n-1} & 0 \\
-I_{2} & 0 & 0
\end{array}\right)
$$

We denote by $\mathbf{P}^{n+2}$ the associated projective space, and by $\mathbf{Q}^{n+1}$ the quadric in $\mathbf{P}^{n+2}$ defined by $\langle x, x\rangle=0$. Then we can identify a Lie sphere in $T_{1} S^{n}$ with a point of $\mathbf{Q}^{n+1}$

Let $\Lambda^{2 n-1}$ be the set of all projective lines on $\mathbf{Q}^{n+1}$. By Line $\{Y, Z\} \in \Lambda^{2 n-1}$ we denote the line generated by $[Y],[Z] \in \mathbf{Q}^{n+1}$. Then

$$
T_{1} S^{n} \cong \Lambda^{2 n-1} \text {. }
$$

A diffeomorphism $\phi: T_{1} S^{n} \rightarrow T_{1} S^{n}$ is called a Lie transformation if it carries Lie spheres to Lie spheres. For example a Möbius transformation and a parallel transformation are Lie transformations; the former takes point spheres to point 
spheres and the latter takes $(\iota, \xi)$ to $(\cos t \iota+\sin t \xi,-\sin t \iota+\cos t \xi)$, where $t$ $\in[0, \pi)$. Lie transformations are generated by Möbius transformations and parallel transformations.

Denote the group of all Lie transformations by $G$. A Lie transformation $\phi$ can be regarded as a diffeomorphism $\phi: \mathbf{Q}^{n+1} \rightarrow \mathbf{Q}^{n+1}$ preserving lines on $\mathbf{Q}^{n+1}$, that is the restriction of a projective transformation $\Phi: \mathbf{P}^{n+2} \rightarrow \mathbf{P}^{n+2}$ preserving $\mathbf{Q}^{n+1}$. Thus,

$$
G \cong P O(n+1,2)=O(n+1,2) /\{ \pm 1\},
$$

where $O(n+1,2)=\left\{P \in G L(n+3 ; \mathbf{R}) ;{ }^{t} P S P=S\right\}$.

Let $o=\left(\mathbf{e}_{1}, \mathbf{e}_{n+1}\right) \in T_{1} S^{n}$ be the origin, where $\left(\mathbf{e}_{1}, \ldots, \mathbf{e}_{n+1}\right)$ is the natural basis of $\mathbf{E}^{n+1}$. The isotropy subgroup $H$ of $G$ at $o$ given by

$$
\begin{gathered}
H=\left\{\left(\begin{array}{ccc}
A & 0 & 0 \\
0 & E & 0 \\
0 & 0 & { }^{t} A^{-1}
\end{array}\right) \exp \left(\begin{array}{ccc}
0 & 0 & 0 \\
B & 0 & 0 \\
0 & { }^{t} B & 0
\end{array}\right) \exp \left(\begin{array}{ccc}
0 & 0 & 0 \\
0 & 0 & 0 \\
C & 0 & 0
\end{array}\right) ;\right. \\
\left.A \in G L(2 ; \mathbf{R}), E \in O(n-1), B \in M_{n-1,2}(\mathbf{R}), C \in \mathfrak{o}(2)\right\} .
\end{gathered}
$$

The group $G$ acts on $\Lambda^{2 n-1}$ transitively, then

$$
\Lambda^{2 n-1} \cong G / H \text {. }
$$

\subsection{Lie frames}

The Lie algebra $\mathfrak{g}$ of $G$ is given by

$$
\begin{aligned}
\mathfrak{g}=\{X & \left.\in \operatorname{gl}(n+3 ; \mathbf{R}) ;{ }^{t} X S+S X=0\right\} \\
= & \left\{\left(\begin{array}{ccc}
\alpha & \delta & \zeta \\
\beta & \varepsilon & { }^{t} \delta \\
\gamma & { }^{t} \beta & -{ }^{t} \alpha
\end{array}\right) ; \begin{array}{c}
\alpha \in M_{2,2}(\mathbf{R}), \beta,{ }^{t} \delta \in M_{n-1,2}(\mathbf{R}), \\
\gamma, \zeta \in \mathcal{o}(2), \varepsilon \in \mathcal{o}(n-1)
\end{array}\right\},
\end{aligned}
$$

and the Lie algebra $\mathfrak{h}$ of $H$ is given by

$$
\mathfrak{h}=\left\{\left(\begin{array}{ccc}
\alpha & 0 & 0 \\
\beta & \varepsilon & 0 \\
\gamma & { }^{t} \beta & -{ }^{t} \alpha
\end{array}\right) ; \begin{array}{c}
\alpha \in M_{2,2}(\mathbf{R}), \beta \in M_{n-1,2}(\mathbf{R}), \\
\gamma \in \mathfrak{o}(2), \varepsilon \in \mathfrak{o}(n-1)
\end{array}\right\} .
$$

A Lie frame $\left(Y_{1}, \ldots, Y_{n+3}\right)$ is an ordered set of vectors in $\mathbf{R}_{2}^{n+3}$ satisfying the relations 


$$
\left\langle Y_{\imath}, Y_{\jmath}\right\rangle=S_{i}
$$

for $1 \leq i, j \leq n+3$. The space of all Lie frames can be identified with $O(n+1$, 2).

Let $\Omega=\left(\omega_{j}^{i}\right)$ be the Maurer-Cartan form introduced by the equation

$$
d Y_{\imath}=\sum_{j=1}^{n+3} \omega_{\imath}^{j} Y_{j}
$$

Taking the exterior derivative of (1.8), we get the Maurer-Cartan equations

$$
d \omega_{i}^{j}=\sum_{j=1}^{n+3} \omega_{i}^{k} \wedge \omega_{k}^{\jmath}
$$

By using (1.8), we find that $\omega_{n+3}^{1} \wedge\left(d \omega_{n+3}^{1}\right)^{n-1} \neq 0$. Hence $T_{1} S^{n}$ is a contact manifold with a contact form $\omega_{n+3}^{1}$.

\subsection{Legendre submanifolds and Dupin submanifolds}

An immersed $(n-1)$-dimensional integral submanifold of the contact distribution $D$ is called a Legendre submanifold.

An immersed hypersurface $f: M^{n-1} \rightarrow S^{n}$ with a unit normal field $\xi: M^{n-1}$ $\rightarrow S^{n}$ naturally induces a Legendre submanifold $\lambda=(f, \xi): M^{n-1} \rightarrow T_{1} S^{n}$. This map $\lambda$ is called the Legendre map induced by $f$ with $\xi$. Conversely a Legendre submanifold $\lambda=(f, \xi): M^{n-1} \rightarrow T_{1} S^{n}$ naturally induces a smooth map $f: M^{n-1} \rightarrow$ $S^{n}$, which may have singularities; however, a Legendre submanifolds is locally transformed by a parallel transformation to be a Legendre map. (See [P Theorem 1].)

Let $Y$ and $Z$ are smooth maps from $M^{n-1}$ into $\mathbf{Q}^{n+1}$. By Line $\{Y(p), Z(p)\}$ we denote the line generated by the points $[Y(p)]$ and $[Z(p)]$ in $\mathbf{Q}^{n+1}$ for $p \in M^{n-1}$. Let $\lambda=\operatorname{Line}\{Y, Z\}: M^{n-1} \rightarrow \Lambda^{2 n-1}$ be a Legendre submanifold. Then,

$$
\langle d Y, Z\rangle=0 .
$$

(For the necessary and sufficient condition that a smooth map $\lambda=\operatorname{Line}\{Y, Z\}$ : $M^{n-1} \rightarrow \Lambda^{2 n-1}$ is a Legendre submanifold, see [C Theorem 2.3].)

We call the sphere

$$
[K(p)]=[r Y(p)+s Z(p)]
$$

a curvature sphere of $\lambda$ at $p \in M^{n-1}$, if there exist a non-zero vector $X$ in $T_{p} M^{n-1}$ and $r, s \in \mathbf{R}$ with $(r, s) \neq(0,0)$ such that

$$
r d Y(X)+s d Z(X) \in \operatorname{Span}\{Y(p), Z(p)\} .
$$


A curvature sphere is invariant under Lie transformations. The vector $X$ is called a principal vector corresponding to $[K]$. At each point $p \in M^{n-1}$, there are at most $n-1$ distinct curvature spheres $\left[K_{1}\right], \ldots,\left[K_{g}\right]$. The principal vectors corresponding to the curvature sphere $\left[K_{\imath}\right]$ form a subspace $T_{\imath}$ of the tangent space $T_{p} M^{n-1}$, and $T_{p} M^{n-1}=T_{1} \oplus \cdots \oplus T_{g}$. If the dimension of $T_{i}$ (which we call the multiplicity of $\left.\left[K_{1}\right]\right)$ is constant on an open subset $U$ of $M^{n-1}$, then the distribution $T_{\imath}$ is integrable on $U$. A connected submanifold $\&$ of $M^{n-1}$ is called a curvature surface if at each $p \in \&$, the tangent space $T_{p} \&$ is equal to a $T_{\imath}$.

A Legendre submanifold $\lambda: M^{n-1} \rightarrow \Lambda^{2 n-1}$ is called a Dupin submanifold if along each curvature surface, the corresponding curvature sphere is constant. We say that a Dupin submanifold is proper if multiplicities of curvature spheres are constant on $M^{n-1}$. If $\&$ is a curvature surface of dimension $m>1$ in a Legendre submanifold, then the corresponding curvature sphere is constant along $\&$. This fact shows that we have only to check the Dupin condition along curvature surfaces with dimension one.

\subsection{The second fundamental form}

Let $\lambda: M^{n-1} \rightarrow \Lambda^{2 n-1}$ be a Legendre submanifold. Let $\left(Y_{1}, \ldots, Y_{n+3}\right)$ be a smooth Lie frame on an open set $U$ of $M^{n-1}$ such that for each $x \in U, \lambda(x)=$ Line $\left\{Y_{n+2}, Y_{n+3}\right\}$. We can choose the Lie frame so that $Y_{n+2}$ is not a curvature sphere at $U$. By (1.4) and (1.9) we find that the forms $\omega_{3}^{1}, \omega_{4}^{1}, \ldots, \omega_{n+1}^{1}$ are linearly independent; i.e.

$$
\omega_{3}^{1} \wedge \omega_{4}^{1} \wedge \cdots \wedge \omega_{n+1}^{1} \neq 0
$$

The condition (1.9) is equal to

$$
\omega_{n+3}^{1}=0 .
$$

Taking the exterior derivative of (1.13) and using the Maurer-Cartan equations (1.8), we obtain that

$$
d \omega_{n+3}^{1}=\sum_{\alpha=3}^{n+1} \omega_{\alpha}^{2} \wedge \omega_{\alpha}^{1}=0
$$

By Cartan's lemma and (1.12) it follows that

$$
\omega_{\alpha}^{2}=\sum_{\beta=3}^{n+1} h_{\alpha \beta} \omega_{\beta}^{1} \quad \text { with } h_{\alpha \beta}=h_{\beta \alpha} .
$$

We define the second fundamental form of $\lambda$ determined by $Y_{n+2}$ to be the quadratic differential form 


$$
I I\left(Y_{n+2}\right)=\sum_{\alpha, \beta=3}^{n+1} h_{\alpha \beta} \omega_{\alpha}^{1} \omega_{\beta}^{1}
$$

\section{Frenet frames}

\subsection{Contact}

Let $N$ be a smooth $n$-dimensional manifold, and let $\lambda: M \rightarrow N$ and $\tilde{\lambda}: \tilde{M} \rightarrow N$ be embedded submanifolds of dimension $m$. We say that $\lambda$ and $\tilde{\lambda}$ have contact of at least order 0 at $p \in M$ and $\tilde{p} \in \tilde{M}$ if $\lambda(p)=\tilde{\lambda}(\tilde{p})$, and that $\lambda$ and $\tilde{\lambda}$ have contact of at least order 1 a $p$ and $\tilde{p}$ if $\lambda(p)=\tilde{\lambda}(\tilde{p})=x$ and $\lambda_{*} M_{p}=\tilde{\lambda}_{*} \tilde{M}_{\tilde{p}}$ as subspaces of $N_{x}$

We reformulate the definition above. Let $G_{n, m}(N)$ denote the Grassmann bundle of tangent $m$-plane on $N$. The immersion $\lambda$ induces the smooth mapping $T_{\lambda}: M$ $\rightarrow G_{n, m}(N)$ given by $T_{\lambda}(p)=\lambda_{*} M_{p}$, which is a $m$-plane in $N_{\lambda(p)}$. Then $\lambda$ and $\tilde{\lambda}$ have contact of at least order 1 at $p$ and $\tilde{p}$ if and only if $T_{\lambda}^{(1)}(p)=T_{\tilde{\lambda}}^{(1)}(\tilde{p})$.

We iterate this construction and define higher order contact as follows: Let $N^{(0)}=N, n_{0}=n$ and for $k \geq 0$ let $N^{(k+1)}=G_{n_{k}, m}\left(N^{(k)}\right)$ where $n_{k+1}=$ $\operatorname{dim} N^{(k+1)}$. Let $T_{\lambda}^{(0)}=\lambda$ and $T_{\lambda}^{(k+1)}=T_{T_{\lambda}^{(k)}}: M \rightarrow G_{n_{k} m}\left(N^{(k)}\right)$. Then for $k \geq 0$ we say that $\lambda$ and $\tilde{\lambda}$ have contact of at least order $k$ at $p$ and $\tilde{p}$ if $T_{\lambda}^{(k)}(p)=T_{\tilde{\lambda}}^{(k)}(\tilde{p})$. If $\lambda$ and $\tilde{\lambda}$ have contact of at least order $k$ at $p \in M$ and $\tilde{p} \in \tilde{M}$ but not of order $k+1$, then we say that $\lambda$ and $\tilde{\lambda}$ have contact of order $k$ at $p$ and $\tilde{p}$.

We say that $\lambda$ and $\tilde{\lambda}$ have $G$-contact of least order $k$ at $\tilde{p}$ if there exists a $P \in$ $G$ such that $\lambda$ and $P \circ \tilde{\lambda}$ have contact of at least order $k$ at $p$ and $\tilde{p}$.

\subsection{Construction of Frenet frames}

Let $G$ be a transitive Lie transformation group on a manifold $N$. We fix an origin $o$ of $N$, and denote the isotropy subgroup of $G$ at $o$ by $H$. Then the map $\pi: G \rightarrow N$ given by $\pi(P)=P(o)$ induces diffeomorphism $G / H \cong N$.

Let $\lambda: M \rightarrow G / H \cong N$ be a connected, smoothly embedded $n$-dimensional submanifold of a homogeneous space $G / H$. Firstly we construct the set of zeroth order frames. A zeroth order frame at $p \in M$ is an element $P \in G$ such that $\pi(P)=\lambda(p)$.

Choosing a basis $e_{1}, \ldots, e_{m_{0}}$ of the tangent space $N_{o}$, we have a natural bundle map $h_{0}: G \rightarrow L(N)$ defined by $h_{0}(P)=P_{*}\left(e_{1}, \ldots, e_{m_{0}}\right)$, where $L(N) \rightarrow N$ is the principal $G L(m, \mathbf{R})$-bundle of linear frames on $N$. We identify $P \in G$ with $h_{0}(P) \in L(N)$, so we call $P$ a frame.

Let $L_{0}$ denote the set of all zeroth order frames on $M$. A zeroth order frame field $u$ along $\lambda$ is a smooth cross section of $L_{0} \rightarrow M$; i.e. is a smooth map $u: M \rightarrow$ 
$G$ such that $\pi \circ u=\lambda$.

Secondly we construct first order frames. We denote by $\mathfrak{g}$ and $\mathfrak{h}$ the Lie algebras of $G$ and $H$ respectively. We take a vector subspace $m$ of $\mathfrak{g}$ complementary to $\mathfrak{h}$, and choose basis $e_{1}, \ldots, e_{m_{0}}$ of $\mathrm{m}$. With respect to this basis, we consider the linear isotropy representation $\rho_{0}: H \rightarrow G L\left(m_{0}, \mathbf{R}\right)$ given by the adjoint action of $H$ on $\mathfrak{g} / \mathfrak{h} \cong \mathfrak{m}$. There is a naturally defined smooth map $\lambda_{0}$ from $L_{0}$ to the Grassmann manifold $G_{m_{0}, n}$ given by $\lambda_{0}(P)=P_{*}^{-1} \lambda_{*} M_{p}$ where $\lambda(p)=\pi(P)$. We choose a local cross section $W_{1}$ of the action $\left(H, \rho_{0}\right)$ on $G_{m_{0}, n}$. We say that $\lambda$ has the type of $W_{1}$ if there exists a zeroth order frame field $u$ along $\lambda$ such that $\lambda_{0}(u) \subset W_{1}$. If $\lambda$ has the type of $W_{1}$, we let $L_{1}=\lambda_{0}^{-1}\left(W_{1}\right)$ and call $L_{1}$ the set of first order frames on $\lambda$ (with respect to $W_{1}$ ). We define a first order frame field along $\lambda$ to be a smooth cross section $L_{1} \rightarrow M$.

The smooth map $\lambda_{0} \circ u: M \rightarrow W_{1}$ does not depend on the choice of first order frame field $u$ along $\lambda$. Choose a coordinate system $x^{1}, \ldots, x^{\mu_{1}}$ on $W_{1}$, where $\mu_{1}=$ $\operatorname{dim} W_{1}$. We call the functions $k^{i}=x^{i} \circ \lambda_{0} \circ u\left(i=1, \ldots, \mu_{1}\right)$ the first order invariants of $\lambda$.

The set of first order frames $L_{1}$ gives first order contact under the action of $G$. To put it more precisely, let $\lambda: M \rightarrow G / H$ and $\tilde{\lambda}: \tilde{M} \rightarrow G / H$ be smoothly embedded $n$-dimensional submanifold on which first order frames can be constructed. Then $\lambda$ and $\tilde{\lambda}$ have $G$-contact of at least order 1 at $p \in M$ and $\tilde{p} \in \tilde{M}$ if and only if they are both the type of a local cross section $W_{1}$ of $\rho_{0}$, and they have the same first order invariants at $p$ and $\tilde{p}$. (See [J 1.6 Theorem 1].)

Furthermore we iterate this construction of frames: $L_{0} \supset L_{1} \supset L_{2} \supset \cdots \supset$ $L_{k} \supset \cdots$. Thus we construct a set of $k$-th order frames $L_{k}$ which gives $k$-th order contact under the action of $G$. The sequence $\operatorname{dim} L_{0} \geq \operatorname{dim} L_{1} \geq \operatorname{dim} L_{2} \geq$ $\cdots$ eventually stabilized. Thus there is the smallest integer $q \geq 1$ such that $\operatorname{dim} L_{k}=\operatorname{dim} L_{q}$ for all $k \geq q$. Then the frames of order $q$ are called the Frenet frames of $\lambda$.

We have the following congruence and existence theorem: Let $\lambda: M \rightarrow G / H$ and $\tilde{\lambda}: \tilde{M} \rightarrow G / H$ be smoothly embedded $n$-dimensional submanifold. Then $\lambda$ and $\tilde{\lambda}$ are $G$-congruent if and only if their Frenet frames are of the same order $q$, they are both the type of a local cross section $W_{q}$ and there exist a one-to-one correspondence $\varphi: M \rightarrow \tilde{M}$ such that $\tilde{k}^{a} \circ \varphi=k^{a}$ where $k^{a}, \tilde{k}^{a}$ are invariants of order $<q$. (See [J I.11 Theorem 3].) 


\section{Lie frames of Legendre maps in $T_{1} S^{3}$ under $P O(4,2)$}

\subsection{Main theorem}

Our main theorem is the following:

THEOREm 3.1. Let $\lambda: M^{2} \rightarrow T_{1} S^{3}$ be a Legendre map which is induced by an embedded oriented surface $f: M^{2} \rightarrow S^{3}$ with a field of unit normals $\xi: M^{2} \rightarrow S^{3}$. Let $\Omega$ be the Maurer-Cartan form on $G=P O(4,2)$ and $\phi_{1}, \phi_{2}$ coframe fields on $M^{2}$. Then the Legendre map $\lambda$ belongs to one of the following fine types.

Type (a): We can take a Lie frame $u: M^{2} \rightarrow G$ of $\lambda$ such that

$$
u^{*} \Omega=\left(\begin{array}{cccccc}
0 & 0 & \phi_{1} & \phi_{2} & 0 & 0 \\
0 & 0 & 0 & 0 & 0 & 0 \\
0 & 0 & 0 & 0 & \phi_{1} & 0 \\
0 & 0 & 0 & 0 & \phi_{2} & 0 \\
0 & 0 & 0 & 0 & 0 & 0 \\
0 & 0 & 0 & 0 & 0 & 0
\end{array}\right)
$$

Type (b): We can take a Lie frame $u: M^{2} \rightarrow G$ of $\lambda$ such that

$$
u^{*} \Omega=\left(\begin{array}{cccccc}
0 & 0 & \phi_{1} & \phi_{2} & 0 & 0 \\
0 & 0 & \phi_{1} & -\phi_{2} & 0 & 0 \\
0 & 0 & 0 & 0 & \phi_{1} & \phi_{1} \\
0 & 0 & 0 & 0 & \phi_{2} & -\phi_{2} \\
0 & 0 & 0 & 0 & 0 & 0 \\
0 & 0 & 0 & 0 & 0 & 0
\end{array}\right)
$$

Type (c): We can take a Lie frame $u: M^{2} \rightarrow G$ of $\lambda$ such that

$$
\begin{aligned}
u^{*} \Omega & =\left(\begin{array}{ccc}
\alpha & \delta & 0 \\
\beta & 0 & { }^{t} \delta \\
\gamma & { }^{t} \beta & -{ }^{t} \alpha
\end{array}\right), \\
\alpha & =\left(\begin{array}{cc}
-3 k^{1} \phi_{1}-3 k^{2} \phi_{2} & k^{1} \phi_{1}+k^{2} \phi_{2} \\
\left(k^{1}+1\right) \phi_{1}+k^{2} \phi_{2} & \left(-3 k^{1}+1\right) \phi_{1}-3 k^{2} \phi_{2}
\end{array}\right), \\
\beta & =\left(\begin{array}{cc}
k^{1} \phi_{1}+\left(k^{2}-k^{3}\right) \phi_{2} & k^{1} \phi_{1}+\left(k^{2}-k^{3}\right) \phi_{2} \\
2 k^{3} \phi_{1}+k^{4} \phi_{2} & -2 k^{3} \phi_{1}-k^{4} \phi_{2}
\end{array}\right), \\
\gamma & k^{4} \phi_{1} \\
\gamma & =\left(\begin{array}{cc}
\phi_{1} & \phi_{2} \\
\phi_{1} & -\phi_{2}
\end{array}\right),
\end{aligned}
$$


In (3.3), $k^{2}(i=1,2,3,4)$ are smooth functions on $M^{2}$ that satisfy the following integrability conditions:

$$
\begin{aligned}
& k^{2}+6 k^{1} k^{3}+k_{1}^{3}=0, \\
& 12 k^{2} k^{3}-8 k^{1} k^{4}+\left(2 k_{2}^{3}-k_{1}^{4}\right)=0 \\
& 8 k^{2} k^{4}+k_{2}^{4}=0, \\
& 2 k^{1} k^{2}+k^{3}-k_{2}^{1}+k_{1}^{2}=0,
\end{aligned}
$$

where $k_{j}^{l}(i=1,2,3,4, j=1,2)$ are smooth functions on $M^{2}$ such that $d k^{2}=k_{1}^{i} \phi_{1}$ $+k_{2}^{i} \phi_{2}$.

Type (d): We can take a Lie frame $u: M^{2} \rightarrow G$ of $\lambda$ such that

$$
\begin{aligned}
u^{*} \Omega= & \left(\begin{array}{ccc}
\alpha & \delta & 0 \\
\beta & 0 & { }^{t} \delta \\
\gamma & { }^{t} \beta & -{ }^{t} \alpha
\end{array}\right), \\
\alpha & =\left(\begin{array}{cc}
3 k^{1} \phi_{1}+3 k^{2} \phi_{2} & k^{1} \phi_{1}+k^{2} \phi_{2} \\
k^{1} \phi_{1}+\left(k^{2}+1\right) \phi_{2} & 3 k^{1} \phi_{1}+\left(3 k^{2}-1\right) \phi_{2}
\end{array}\right), \\
\beta & =\left(\begin{array}{cc}
k^{4} \phi_{1}-2 k^{3} \phi_{2} & k^{4} \phi_{1}-2 k^{3} \phi_{2} \\
\left(k^{1}+k^{3}\right) \phi_{1}+k^{2} \phi_{2} & \left(-k^{1}-k^{3}\right) \phi_{1}-k^{2} \phi_{2}
\end{array}\right), \\
\gamma & =\left(\begin{array}{cc}
0 & k^{4} \phi_{2} \\
-k^{4} \phi_{2} & 0
\end{array}\right), \quad \delta=\left(\begin{array}{cc}
\phi_{1} & \phi_{2} \\
\phi_{1} & -\phi_{2}
\end{array}\right) .
\end{aligned}
$$

In (3.5), $k^{2}(i=1,2,3,4)$ are smooth functions on $M^{2}$ that satisfy the following integrability conditions:

$$
\begin{aligned}
& k^{1}-6 k^{2} k^{3}+k_{2}^{3}=0, \\
& 12 k^{1} k^{3}+8 k^{2} k^{4}+\left(-2 k_{1}^{3}-k_{2}^{4}\right)=0 \\
& 8 k^{1} k^{4}-k_{1}^{4}=0 \\
& 2 k^{1} k^{2}-k^{3}-k_{2}^{1}+k_{1}^{2}=0,
\end{aligned}
$$

where $k_{j}^{2}(i=1,2,3,4, j=1,2)$ are smooth functions on $M^{2}$ such that $d k^{2}=k_{1}^{2} \phi_{1}+$ $k_{2}^{i} \phi_{2}$.

Type (e): We can take a Lie frame $u: M^{2} \rightarrow G$ of $\lambda$ such that

$$
u^{*} \Omega=\left(\begin{array}{ccc}
\alpha & \delta & 0 \\
\beta & 0 & { }^{t} \delta \\
\gamma & { }^{t} \beta & -{ }^{t} \alpha
\end{array}\right),
$$




$$
\begin{aligned}
& \alpha=\left(\begin{array}{cc}
\left(3 k^{1}+1\right) \phi_{1}-\left(3 k^{2}+1\right) \phi_{2} & k^{1} \phi_{1}+k^{2} \phi_{2} \\
\left(k^{1}+1\right) \phi_{1}+\left(k^{2}+1\right) \phi_{2} & \left(3 k^{1}+2\right) \phi_{1}-\left(3 k^{2}+2\right) \phi_{2}
\end{array}\right), \\
& \beta=\left(\begin{array}{cc}
k^{5} \phi_{1}+k^{3} \phi_{2} & k^{5} \phi_{1}+k^{3} \phi_{2} \\
k^{4} \phi_{1}+k^{6} \phi_{2} & -k^{4} \phi_{1}-k^{6} \phi_{2}
\end{array}\right), \\
& \gamma=\left(\begin{array}{cc}
0 & k^{6} \phi_{1}+k^{5} \phi_{2} \\
-k^{6} \phi_{1}-k^{5} \phi_{2} & 0
\end{array}\right), \quad \delta=\left(\begin{array}{cc}
\phi_{1} & \phi_{2} \\
\phi_{1} & -\phi_{2}
\end{array}\right) .
\end{aligned}
$$

In (3.7), $k^{i}(i=1, \ldots, 6)$ are smooth functions on $M^{2}$ that satisfy the following integrability conditions:

$$
\begin{aligned}
& k^{3}=\left(k^{1}+k^{2}\right)^{2}+2 k_{2}^{1}+k_{1}^{2}, \\
& k^{4}=\left(k^{1}+k^{2}\right)^{2}-k_{2}^{1}+2 k_{1}^{2}, \\
& 6 k^{1} k^{3}+4 k^{2} k^{5}+3 k^{3}+2 k^{5}-k_{1}^{3}+k_{2}^{5}=0, \\
& 4 k^{1} k^{6}+6 k^{2} k^{4}+3 k^{4}+2 k^{6}+k_{2}^{4}-k_{1}^{6}=0 \\
& 4 k^{1} k^{5}+4 k^{2} k^{6}+2 k^{5}+2 k^{6}+k_{1}^{5}-k_{2}^{6}=0,
\end{aligned}
$$

where $k_{j}^{2}(i=1, \ldots, 6, j=1,2)$ are smooth functions on $M^{2}$ such that $d k^{2}=k_{1}^{2} \phi_{1}$ $+k_{2}^{i} \phi_{2}$.

\subsection{Proof of Theorem 3.1}

Before turning to the proof of Theorem 3.1, we indicate its process in Figure 1.

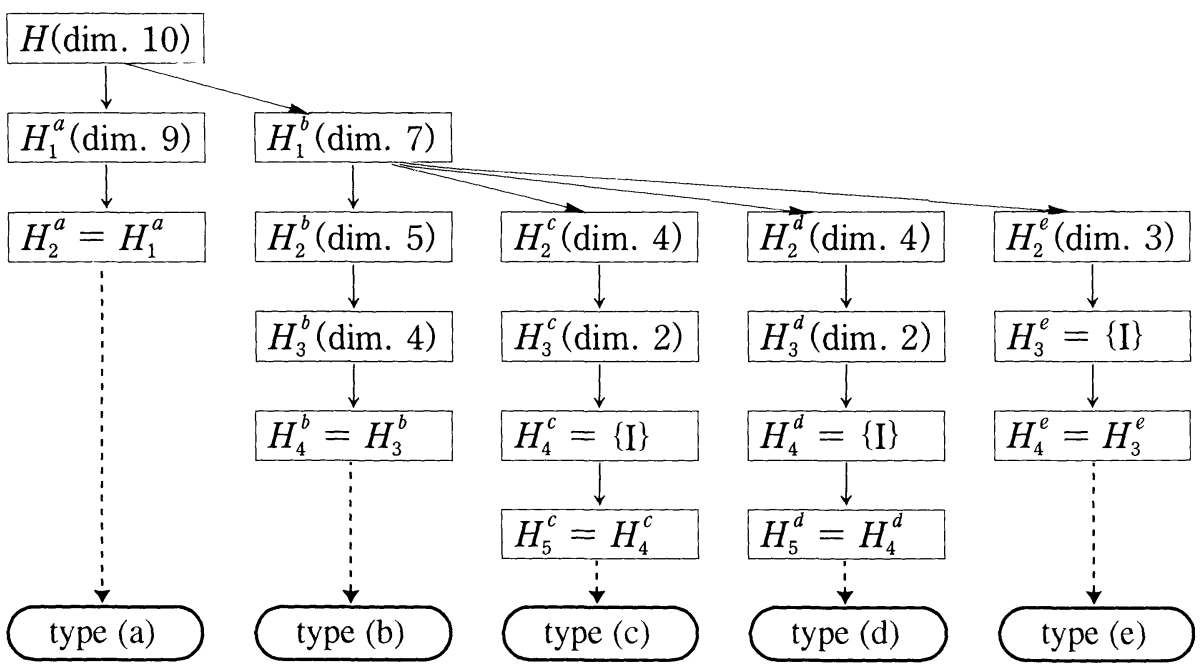

Figure 1 
The detail of the proof of Theorem 3.1 is as follows:

\section{Construction of first order frames}

We denote by $H$ the isotropy subgroup of $G$ at the origin $o$, and $\mathfrak{g}$ and $\mathfrak{h}$ the Lie algebra of $G$ and $H$ respectively. (See (1.3), (1.4) and (1.5).) As a vector subspace of $\mathfrak{g}$ complementary to $\mathfrak{h}$, we take the $\operatorname{Ad}(H)$-invariant subspace $\mathfrak{m}$. For a basis of $\mathrm{m}$ we choose the following $e_{1}, e_{2}, e_{3}, e_{4}, e_{5}$ :

$$
\begin{aligned}
& e_{1}=\left(\begin{array}{ccc}
0 & 0 & E_{1} \\
0 & 0 & 0 \\
0 & 0 & 0
\end{array}\right), e_{2}=\left(\begin{array}{ccc}
0 & E_{2} & 0 \\
0 & 0 & { }^{t} E_{2} \\
0 & 0 & 0
\end{array}\right), e_{3}=\left(\begin{array}{ccc}
0 & E_{3} & 0 \\
0 & 0 & { }^{t} E_{3} \\
0 & 0 & 0
\end{array}\right), \\
& e_{4}=\left(\begin{array}{ccc}
0 & E_{4} & 0 \\
0 & 0 & { }^{t} E_{4} \\
0 & 0 & 0
\end{array}\right), e_{5}=\left(\begin{array}{ccc}
0 & E_{5} & 0 \\
0 & 0 & { }^{t} E_{5} \\
0 & 0 & 0
\end{array}\right),
\end{aligned}
$$

where

$$
E_{1}=\left(\begin{array}{cc}
0 & 1 \\
-1 & 0
\end{array}\right), E_{2}=\left(\begin{array}{ll}
1 & 0 \\
0 & 0
\end{array}\right), E_{3}=\left(\begin{array}{ll}
0 & 1 \\
0 & 0
\end{array}\right), E_{4}=\left(\begin{array}{ll}
0 & 0 \\
1 & 0
\end{array}\right), E_{5}=\left(\begin{array}{ll}
0 & 0 \\
0 & 1
\end{array}\right)
$$

The isotropy representation $\rho_{0}: H \rightarrow G L(5, \mathbf{R})$ is

$$
\rho_{0}(P)=\left(\begin{array}{ccc}
\operatorname{det} A & 0 & 0 \\
* & a_{1}^{1} E & a_{2}^{1} E \\
* & a_{1}^{1} E & a_{2}^{2} E
\end{array}\right)=\left(\begin{array}{ccc}
\operatorname{det} A & 0 & 0 \\
* & a_{1}^{1} I_{2} & a_{2}^{1} I_{2} \\
* & a_{1}^{2} I_{2} & a_{2}^{2} I_{2}
\end{array}\right)\left(\begin{array}{ccc}
1 & 0 & 0 \\
* & E & 0 \\
* & 0 & E
\end{array}\right)
$$

for $P \in H, A=\left(\begin{array}{ll}a_{1}^{1} & a_{2}^{1} \\ a_{1}^{2} & a_{2}^{2}\end{array}\right) \in G L(2, \mathbf{R}), E \in O(2)$. Let $\bar{A}$ denote the former matrix determined by $A$ in (3.11), and $\bar{E}$ the latter matrix determined by $E$ in (3.11). With respect to $\mathfrak{g}=\mathfrak{h}+\mathfrak{m}$ we decompose the Maurer-Cartan form $\Omega$ into $\Omega_{0}+\Theta_{0}$, and we set $\Theta_{0}=\sum \theta_{\imath} e_{\imath}(i=1,2,3,4,5)$ then

$$
\theta_{1}=\omega_{6}^{1}, \theta_{2}=\omega_{3}^{1}, \theta_{2}=\omega_{4}^{1}, \theta_{4}=\omega_{3}^{2}, \theta_{5}=\omega_{4}^{2} .
$$

We need to choose a local cross section $W_{1}$ of the action $\left(H, \rho_{0}\right)$ on $G_{5,2}$ to obtain a first order frames on $\lambda$. To get orbits of the action of $\left(H, \rho_{0}\right)$, we consider the Maurer-Cartan form $\Omega$ on G. Let $u$ be a zeroth order frame field along $\lambda$, and $\phi_{1}, \phi_{2}$ coframe fields on $M$. Set $u^{*} \theta_{\imath}=x_{\imath} \phi_{1}+y_{i} \phi_{2}$ for some smooth functions $x_{\imath}, y_{\imath}$ on $M$, then 


$$
\lambda_{0}(u)=\left[\begin{array}{ll}
x_{1} & y_{1} \\
x_{2} & y_{2} \\
x_{3} & y_{3} \\
x_{4} & y_{4} \\
x_{5} & y_{5}
\end{array}\right]: M \rightarrow G_{5,2}
$$

(For further details, see [J].) Hence $u$ is a first order frame field along $\lambda$ with respect to $W_{1}$ if and only if

$$
{ }^{t}\left[\begin{array}{lllll}
x_{1} & x_{2} & x_{3} & x_{4} & x_{5} \\
y_{1} & y_{2} & y_{3} & y_{4} & y_{5}
\end{array}\right] \in W_{1}
$$

PROPOSITION 3.2. We can take two types of $W_{1}$ :

$$
W_{1}^{a}=\left\{{ }^{t}\left[\begin{array}{lllll}
0 & 1 & 0 & 0 & 0 \\
0 & 0 & 1 & 0 & 0
\end{array}\right]\right\}, \quad W_{1}^{b}=\left\{{ }^{t}\left[\begin{array}{lllcc}
0 & 1 & 0 & 1 & 0 \\
0 & 0 & 1 & 0 & -1
\end{array}\right]\right\} .
$$

Proof. We see that $x_{1}=y_{1}=0$, because $\lambda$ is a Legendre map and $\omega_{6}^{1}$ is a contact form. We choose coframe fields $\phi_{1}, \phi_{2}$ on $M$ such that $u^{*} \theta_{2}=\phi_{1}, u^{*} \theta_{3}=$ $\phi_{2}$. From (1.15),

$$
\left[\begin{array}{ll}
x_{1} & y_{1} \\
x_{2} & y_{2} \\
x_{3} & y_{3} \\
x_{4} & y_{4} \\
x_{5} & y_{5}
\end{array}\right]=\left[\begin{array}{cc}
0 & 0 \\
1 & 0 \\
0 & 1 \\
h_{33} & h_{34} \\
h_{43} & h_{44}
\end{array}\right]=\left[\begin{array}{l}
0 \\
I_{2} \\
H
\end{array}\right]
$$

We first consider the orbit of the action of $\bar{E}$, where $E \in O(2)$.

$$
\bar{E}\left[\begin{array}{c}
0 \\
I_{2} \\
H
\end{array}\right]=\left[\begin{array}{c}
0 \\
E \\
E H
\end{array}\right]=\left[\begin{array}{c}
0 \\
I_{2} \\
E H E^{-1}
\end{array}\right] .
$$

Thus those orbits meet ${ }^{t}\left[\begin{array}{lllcc}0 & 1 & 0 & \lambda_{1} & 0 \\ 0 & 0 & 1 & 0 & \lambda_{2}\end{array}\right]$.

Next we consider the orbit of the action of $\bar{A}$, where $A \in G L(2, \mathbf{R})$. 


$$
\bar{A}\left[\begin{array}{cc}
0 & 0 \\
1 & 0 \\
0 & 1 \\
\lambda_{1} & 0 \\
0 & \lambda_{2}
\end{array}\right]=\left[\begin{array}{cc}
0 & 0 \\
a_{1}^{1}+\lambda_{1} a_{2}^{1} & 0 \\
0 & a_{1}^{1}+\lambda_{2} a_{2}^{1} \\
a_{1}^{2}+\lambda_{1} a_{2}^{2} & 0 \\
0 & a_{1}^{2}+\lambda_{2} a_{2}^{2}
\end{array}\right]=\left[\begin{array}{cc}
0 & 0 \\
1 & 0 \\
0 & 1 \\
\frac{a_{1}^{2}+\lambda_{1} a_{2}^{2}}{a_{1}^{1}+\lambda_{1} a_{2}^{1}} & 0 \\
0 & \frac{a_{1}^{2}+\lambda_{2} a_{2}^{2}}{a_{1}^{1}+\lambda_{2} a_{2}^{1}}
\end{array}\right] .
$$

In case that $\lambda_{1}=\lambda_{2}$, those orbits meet ${ }^{t}\left[\begin{array}{lllll}0 & 1 & 0 & 0 & 0 \\ 0 & 0 & 1 & 0 & 0\end{array}\right]$, and in case that $\lambda_{1} \neq$ $\lambda_{2}$, those orbits meet ${ }^{t}\left[\begin{array}{llllc}0 & 1 & 0 & 1 & 0 \\ 0 & 0 & 1 & 0 & -1\end{array}\right]$.

We say that $\lambda: M \rightarrow T_{1} S^{3}$ is of type (a) if it has the type of $W_{1}^{a}$, and that $\lambda$ is of type (B) if it has the type of $W_{1}^{b}$.

\section{Type (a)}

Let $\lambda: M \rightarrow T_{1} S^{3}$ be of type (a); i.e. there exists a zeroth order frame field $u$ along $\lambda$ such that

$$
\begin{aligned}
& u^{*} \omega_{6}^{1}=u^{*} \theta_{1}=0, u^{*} \omega_{3}^{1}=u^{*} \theta_{1}=\phi_{1}, u^{*} \omega_{4}^{1}=u^{*} \theta_{3}=\phi_{2}, \\
& u^{*} \omega_{3}^{2}=u^{*} \theta_{4}=0, u^{*} \omega_{4}^{2}=u^{*} \theta_{5}=0 .
\end{aligned}
$$

Construction of second order frames of type (a)

The isotropy subgroup $H_{1}^{a}$ of $H$ at a point of $W_{1}^{a}$ is

$$
\begin{gathered}
H_{1}^{a}=\left\{\left(\begin{array}{ccc}
A & 0 & 0 \\
0 & E & 0 \\
0 & 0 & { }^{t} A^{-1}
\end{array}\right) \exp \left(\begin{array}{ccc}
0 & 0 & 0 \\
B & 0 & 0 \\
0 & { }^{t} B & 0
\end{array}\right) \exp \left(\begin{array}{ccc}
0 & 0 & 0 \\
0 & 0 & 0 \\
C & 0 & 0
\end{array}\right)\right. \\
\left.A=\left(\begin{array}{cc}
a_{1}^{1} & a_{2}^{1} \\
0 & a_{2}^{2}
\end{array}\right) \in G L(2, \mathbf{R}), E \in O(2), B \in M_{2,2}(\mathbf{R}), C \in \mathfrak{o}(2)\right\} .
\end{gathered}
$$

The Lie algebra $\mathfrak{h}_{1}^{a}$ of $H_{1}^{a}$ is

$$
\mathfrak{h}_{1}^{a}=\left\{\left(\begin{array}{ccc}
\alpha & 0 & 0 \\
\beta & \varepsilon & 0 \\
\gamma & { }^{t} \beta & -{ }^{t} \alpha
\end{array}\right) ; \alpha=\left(\begin{array}{cc}
\alpha_{1}^{1} & \alpha_{2}^{1} \\
0 & \alpha_{2}^{2}
\end{array}\right), \beta \in M_{2,2}(\mathbf{R}), \gamma, \varepsilon \in{ }^{o}(2)\right\} .
$$


We decompose $\mathfrak{h}=\mathfrak{h}_{1}^{a}+\mathfrak{m}_{1}^{a}$, and for a basis of $\mathfrak{m}_{1}^{a}$ we take

$$
e_{6}=\left(\begin{array}{ccc}
E_{4} & 0 & 0 \\
0 & 0 & 0 \\
0 & 0 & -{ }^{t} E_{4}
\end{array}\right) \text {. }
$$

The representation $\rho_{1}^{a}: H_{1}^{a} \rightarrow G L(6, \mathbf{R})$ is

$$
\rho_{1}^{a}(P)=\left(\begin{array}{cccccc}
* & 0 & 0 & * & * & 0 \\
* & & & * & * & 0 \\
* & a_{1}^{1} E & * & * & 0 \\
* & 0 & 0 & * & * & 0 \\
* & 0 & 0 & * & * & 0 \\
* & 0 & 0 & * & * & \frac{a_{2}^{2}}{a_{1}^{1}}
\end{array}\right)
$$

where $P \in H_{1}^{a}$. With respect to $\mathfrak{h}=\mathfrak{h}_{1}^{a}+\mathfrak{m}_{1}^{a}$ we have $\Omega_{0}=\Omega_{1}^{a}+\Theta_{1}^{a}$, and we set $\Theta_{1}^{a}=\theta_{6}^{a} e_{6}$ then

$$
\theta_{6}^{a}=\omega_{1}^{2}
$$

Taking the exterior derivative of (3.15a), and using (3.14) and the Maurer-Cartan equation (1.8), we obtain the following equations:

$$
d u^{*} \theta_{4}=\phi_{1} \wedge u^{*} \theta_{6}^{a}=0, \quad d u^{*} \theta_{5}=\phi_{2} \wedge u^{*} \theta_{6}^{a}=0,
$$

where $u$ is a first order frame field along $\lambda$. It follows that

$$
u^{*} \theta_{6}^{a}=0 \text {. }
$$

As a result, we take a local cross section $W_{2}^{a}=\left\{{ }^{t}\left[\begin{array}{llllll}0 & 1 & 0 & 0 & 0 & 0 \\ 0 & 0 & 1 & 0 & 0 & 0\end{array}\right]\right\}$ of the action $\left(H_{1}^{a}, \rho_{1}^{a}\right)$ on $G_{6,2}$. For this reason, a first order frame field $u$ along $\lambda$ is a second order frame field along $\lambda$ with respect to $W_{2}^{a}$; i.e.

$$
u^{*}\left(\Theta_{0}+\Theta_{1}^{a}\right)=\phi_{1} e_{2}+\phi_{2} e_{3} .
$$

The isotropy subgroup $H_{2}^{a}$ of $H_{1}^{a}$ at a point of $W_{2}^{a}$ is equal to $H_{1}^{a}$. In this way we get a second order frame field $u: M \rightarrow G / H_{1}^{a}$ along $\lambda: M \rightarrow G / H$. Adding an extra step we lift $u$ from $G / H_{1}^{a}$ to $G$, then we get a Lie frame of $\lambda$. By choosing a lifting $\tilde{u}$ of $u$ such that $\tilde{u}^{*} \Omega_{1}^{a}=0$, we obtain the Lie frame of type (a) in Theorem 3.1 . 


\section{Type (B)}

Let $\lambda: M \rightarrow T_{1} S^{3}$ be of type (B); i.e. there exists a zeroth order frame field $u$ along $\lambda$ which satisfies (3.14) and the following equations:

$$
u^{*} \omega_{3}^{2}=u^{*} \theta_{4}=\phi_{1}, \quad u^{*} \omega_{4}^{2}=u^{*} \theta_{5}=-\phi_{2} .
$$

Construction of second order frames of type (B)

The isotropy subgroup $H_{1}^{b}$ of $H$ at a point of $W_{1}^{b}$ is

$$
\begin{gathered}
H_{1}^{b}=\left\{\left(\begin{array}{ccc}
A & 0 & 0 \\
0 & \pm I_{2} & 0 \\
0 & 0 & { }^{t} A^{-1}
\end{array}\right) \exp \left(\begin{array}{ccc}
0 & 0 & 0 \\
B & 0 & 0 \\
0 & { }^{t} B & 0
\end{array}\right) \exp \left(\begin{array}{ccc}
0 & 0 & 0 \\
0 & 0 & 0 \\
C & 0 & 0
\end{array}\right)\right. \\
\left.A=\left(\begin{array}{ll}
a_{1} & a_{2} \\
a_{2} & a_{1}
\end{array}\right) \in G L(2, \mathbf{R}), B \in M_{2,2}(\mathbf{R}), C \in \mathrm{o}(2)\right\}
\end{gathered}
$$

The Lie algebra $\mathfrak{h}_{1}^{b}$ of $H_{1}^{b}$ is

$$
\mathfrak{h}_{1}^{b}=\left\{\left(\begin{array}{ccc}
\alpha & 0 & 0 \\
\beta & 0 & 0 \\
\gamma & { }^{t} \beta & -{ }^{t} \alpha
\end{array}\right) ; \alpha=\left(\begin{array}{cc}
\alpha_{1} & \alpha_{2} \\
\alpha_{2} & \alpha_{1}
\end{array}\right), \beta \in M_{2,2}(\mathbf{R}), \gamma \in \mathfrak{o}(2)\right\} .
$$

We decompose $\mathfrak{h}=\mathfrak{h}_{1}^{b}+\mathfrak{m}_{1}^{b}$, and for a basis of $\mathfrak{m}_{1}^{b}$ we take

$$
e_{6}=\left\{\left(\begin{array}{ccc}
E_{4} & 0 & 0 \\
0 & 0 & 0 \\
0 & 0 & -{ }^{t} E_{4}
\end{array}\right), e_{7}=\left(\begin{array}{ccc}
E_{5} & 0 & 0 \\
0 & 0 & 0 \\
0 & 0 & -{ }^{t} E_{5}
\end{array}\right), e_{8}=\left(\begin{array}{ccc}
0 & 0 & 0 \\
0 & E_{1} & 0 \\
0 & 0 & 0
\end{array}\right)\right. \text {. }
$$

The representation $\rho_{1}^{b}: H_{1}^{b} \rightarrow G L(8, \mathbf{R})$ is

$$
\left(\begin{array}{cccccccc}
\rho_{1}^{b}(P) & 0 & 0 & 0 & 0 & 0 & 0 \\
* & a_{1} & 0 & a_{2} & 0 & 0 & 0 & 0 \\
* & 0 & a_{1} & 0 & a_{2} & 0 & 0 & 0 \\
* & a_{2} & 0 & a_{1} & 0 & 0 & 0 & 0 \\
* & 0 & a_{2} & 0 & a_{1} & 0 & 0 & 0 \\
* & p b_{2}^{1}-q b_{1}^{1} & p b_{2}^{2}-q b_{1}^{2} & -p b_{1}^{1}+q b_{2}^{1} & -p b_{1}^{2}+q b_{2}^{2} & p & -q & 0 \\
* & -q b_{2}^{1}+p b_{1}^{1} & -q b_{2}^{2}+p b_{1}^{2} & q b_{1}^{1}-p b_{2}^{1} & q b_{1}^{2}-q b_{2}^{2} & -q & p & 0 \\
* & -b_{1}^{2} & b_{1}^{1} & -b_{2}^{2} & b_{2}^{1} & 0 & 0 & 1
\end{array}\right)
$$




$$
=\left(\begin{array}{cccccccc}
* & 0 & 0 & 0 & 0 & 0 & 0 & 0 \\
* & a_{1} & 0 & a_{2} & 0 & 0 & 0 & 0 \\
* & 0 & a_{1} & 0 & a_{2} & 0 & 0 & 0 \\
* & a_{2} & 0 & a_{1} & 0 & 0 & 0 & 0 \\
* & 0 & a_{2} & 0 & a_{1} & 0 & 0 & 0 \\
* & 0 & 0 & 0 & 0 & p & -q & 0 \\
* & 0 & 0 & 0 & 0 & -q & p & 0 \\
* & 0 & 0 & 0 & 0 & 0 & 0 & 1
\end{array}\right)\left(\begin{array}{cccccccc}
* & 0 & 0 & 0 & 0 & 0 & 0 & 0 \\
* & 1 & 0 & 0 & 0 & 0 & 0 & 0 \\
* & 0 & 1 & 0 & 0 & 0 & 0 & 0 \\
* & 0 & 0 & 1 & 0 & 0 & 0 & 0 \\
* & 0 & 0 & 0 & 1 & 0 & 0 & 0 \\
* & b_{2}^{1} & b_{2}^{2} & -b_{1}^{1} & -b_{1}^{2} & 1 & 0 & 0 \\
* & b_{1}^{1} & b_{1}^{2} & -b_{2}^{1} & -b_{2}^{2} & 0 & 1 & 0 \\
* & -b_{1}^{2} & b_{1}^{1} & -b_{2}^{2} & b_{2}^{1} & 0 & 0 & 1
\end{array}\right)
$$

where $P \in H_{1}^{b}, p=\frac{\left(a_{1}\right)^{2}+\left(a_{2}\right)^{2}}{\operatorname{det} A}, q=\frac{2 a_{1} a_{2}}{\operatorname{det} A}$. Let $\tilde{A}$ denote the former matrix determined by $A$, and $\tilde{B}$ the latter matrix determined by $B$. With respect to $\mathfrak{h}=\mathfrak{h}_{1}^{b}$ $+\mathrm{m}_{1}^{b}$ we have $\Omega_{0}=\Omega_{1}^{b}+\Theta_{1}^{b}$, and we set $\Theta_{1}^{b}=\sum \theta_{i}^{b} e_{\imath}^{b}(i=6,7,8)$ then

$$
\theta_{6}^{b}=\omega_{1}^{2}-\omega_{2}^{1}, \quad \theta_{7}^{b}=\omega_{2}^{2}-\omega_{1}^{1}, \quad \theta_{8}^{b}=\omega_{4}^{3} .
$$

By taking the exterior derivative of (3.15b), and by using (3.14) and the Maurer-Cartan equation (1.8), we obtain the following equations:

$$
\begin{aligned}
& d\left(\phi_{1}-u^{*} \theta_{4}\right)=-\phi_{1} \wedge u^{*} \theta_{6}^{b}-\phi_{1} \wedge u^{*} \theta_{7}^{b}-2 u^{*} \theta_{8}^{b} \wedge \phi_{2}=0, \\
& d\left(\phi_{2}+u^{*} \theta_{5}\right)=-u^{*} \theta_{6}^{b} \wedge \phi_{2}+u^{*} \theta_{7}^{b} \wedge \phi_{2}-2 \phi_{1} \wedge u^{*} \theta_{8}^{b}=0 .
\end{aligned}
$$

Set $u^{*} \theta_{i}^{b}=x_{i} \phi_{1}+y_{\imath} \phi_{2}(i=6,7,8)$ for some smooth functions $x_{\imath}, y_{\imath}$ on $M$. From (3.21b),

$$
y_{6}+y_{7}+2 x_{8}=0, \quad x_{6}-y_{7}+2 y_{8}=0
$$

We put

$$
x_{6}+y_{8}=x_{7}-x_{8}=X, \quad y_{6}+x_{8}=-y_{7}-x_{8}=Y .
$$

We consider orbits and local sections $W_{2}$ of the action of $\left(H_{1}^{6}, \rho_{1}^{b}\right)$ on $G_{8,2}$.

Proposition 3.3. We can take four types of $W_{2}$ :

$$
\begin{aligned}
& W_{2}^{b}=\left\{{ }^{t}\left[\begin{array}{llllclll}
0 & 1 & 0 & 1 & 0 & 0 & 0 & 0 \\
0 & 0 & 1 & 0 & -1 & 0 & 0 & 0
\end{array}\right]\right\}, \\
& W_{2}^{c}=\left\{{ }^{t}\left[\begin{array}{llllclll}
0 & 1 & 0 & 1 & 0 & 1 & 1 & 0 \\
0 & 0 & 1 & 0 & -1 & 0 & 0 & 0
\end{array}\right]\right\}, \\
& W_{2}^{d}=\left\{{ }^{t}\left[\begin{array}{llllclcl}
0 & 1 & 0 & 1 & 0 & 0 & 0 & 0 \\
0 & 0 & 1 & 0 & -1 & 1 & -1 & 0
\end{array}\right]\right\}, \\
& W_{2}^{e}=\left\{{ }^{t}\left[\begin{array}{llllclcl}
0 & 1 & 0 & 1 & 0 & 1 & 1 & 0 \\
0 & 0 & 1 & 0 & -1 & 1 & -1 & 0
\end{array}\right]\right\},
\end{aligned}
$$


Proof. We first consider the orbit of the action of $\tilde{B}$, where $B \in M_{2,2}(\mathbf{R})$.

$$
\tilde{B}\left[\begin{array}{cc}
0 & 0 \\
1 & 0 \\
0 & 1 \\
1 & 0 \\
0 & -1 \\
x_{6} & y_{6} \\
x_{7} & y_{7} \\
x_{8} & y_{8}
\end{array}\right]=\left[\begin{array}{cc}
0 & 0 \\
1 & 0 \\
0 & 1 \\
1 & 0 \\
0 & -1 \\
-\left(b_{1}^{1}-b_{2}^{1}\right)+x_{6} & \left(b_{1}^{2}+b_{2}^{2}\right)+y_{6} \\
\left(b_{1}^{1}-b_{2}^{1}\right)+x_{7} & \left(b_{1}^{2}+b_{2}^{2}\right)+y_{7} \\
-\left(b_{1}^{2}+b_{2}^{2}\right)+x_{8} & \left(b_{1}^{1}-b_{2}^{1}\right)+y_{8}
\end{array}\right]
$$

Thus those orbits meet ${ }^{t}\left[\begin{array}{cccccccc}0 & 1 & 0 & 1 & 0 & X & X & 0 \\ 0 & 0 & 1 & 0 & -1 & Y & -Y & 0\end{array}\right]$.

Next we consider the orbit of the action of $\tilde{A}$, where $A=\left(\begin{array}{ll}a_{1} & a_{2} \\ a_{2} & a_{1}\end{array}\right) \in G L(2, \mathbf{R})$.

$$
\tilde{A}\left[\begin{array}{cc}
0 & 0 \\
1 & 0 \\
0 & 1 \\
1 & 0 \\
0 & -1 \\
X & Y \\
X & -Y \\
0 & 0
\end{array}\right]=\left[\begin{array}{cc}
0 & 0 \\
a_{1}+a_{2} & 0 \\
0 & a_{1}-a_{2} \\
a_{1}+a_{2} & 0 \\
0 & -a_{1}+a_{2} \\
(p-q) X & (p+q) Y \\
(p-q) X & -(p+q) Y \\
0 & 0
\end{array}\right]=\left[\begin{array}{cc}
0 & 0 \\
1 & 0 \\
0 & 1 \\
1 & 0 \\
0 & -1 \\
\frac{p-q}{a_{1}+a_{2}} X & \frac{p+q}{a_{1}-a_{2}} Y \\
\frac{p-q}{a_{1}+a_{2}} X & -\frac{p+q}{a_{1}-a_{2}}-Y \\
0 & 0
\end{array}\right]
$$

In case that $X=0, Y=0$, those orbits meet the point of $W_{2}^{b}$, in case that $X \neq 0$, $Y=0$, those orbits meet the point of $W_{2}^{c}$, in case that $X=0, Y \neq 0$, those orbits meet the point of $W_{2}^{d}$ and in case that $X \neq 0, Y \neq 0$, those orbits meet the point of $W_{2}^{e}$.

We say that $\lambda: M \rightarrow T_{1} S^{3}$ is of type (B-b) if it has the type of $W_{2}^{b}$. (We abbreviate "type (B-b)" to "type (b)".) And we say that $\lambda$ is type (c), type (d) or type (e) if it has the type or $W_{2}^{c}, W_{2}^{d}$ or $W_{2}^{e}$ respectively.

\section{Type (b)}

Let $\lambda: M \rightarrow T_{1} S^{3}$ be of type (b); i.e. there exists a first order frame field $u$ along $\lambda$ which satisfies (3.14), (3.15b) and the following equations: 
$(3.23 b)$

$u^{*}\left(\omega_{1}^{2}-\omega_{2}^{1}\right)=u^{*} \theta_{6}^{b}=0, u^{*}\left(\omega_{2}^{2}-\omega_{1}^{1}\right)=u^{*} \theta_{7}^{b}=0, u^{*} \omega_{4}^{3}=u^{*} \theta_{8}^{b}=0$.

Construction of third order frames of type (b)

The isotropy subgroup $H_{2}^{b}$ of $H_{1}^{b}$ at a point of $W_{2}^{b}$ is

$$
\begin{gathered}
H_{2}^{b}=\left\{\left(\begin{array}{ccc}
A & 0 & 0 \\
0 & I_{2} & 0 \\
0 & 0 & { }^{t} A^{-1}
\end{array}\right) \exp \left(\begin{array}{ccc}
0 & 0 & 0 \\
B & 0 & 0 \\
0 & { }^{t} B & 0
\end{array}\right) \exp \left(\begin{array}{ccc}
0 & 0 & 0 \\
0 & 0 & 0 \\
C & 0 & 0
\end{array}\right) ;\right. \\
\left.A=\left(\begin{array}{ll}
a_{1} & a_{2} \\
a_{2} & a_{1}
\end{array}\right) \in G L(2, \mathbf{R}), B=\left(\begin{array}{cc}
b_{1} & b_{1} \\
b_{2} & -b_{2}
\end{array}\right), C \in \mathfrak{o}(2)\right\} .
\end{gathered}
$$

The Lie algebra $\mathfrak{h}_{2}^{b}$ of $H_{2}^{b}$ is

$$
\mathfrak{h}_{2}^{b}=\left\{\left(\begin{array}{ccc}
\alpha & 0 & 0 \\
\beta & 0 & 0 \\
\gamma & { }^{t} \beta & -{ }^{t} \alpha
\end{array}\right) ; \alpha=\left(\begin{array}{cc}
\alpha_{1} & \alpha_{2} \\
\alpha_{2} & \alpha_{1}
\end{array}\right), \beta=\left(\begin{array}{cc}
\beta_{1} & \beta_{1} \\
\beta_{2} & -\beta_{2}
\end{array}\right), \gamma \in \mathfrak{o}(2)\right\} \text {. }
$$

We decompose $\mathfrak{h}_{1}^{b}=\mathfrak{h}_{2}^{b}+\mathfrak{m}_{2}^{b}$, and for a basis of $\mathfrak{m}_{2}^{b}$ we take

$$
e_{9}^{b}=\left\{\left(\begin{array}{ccc}
0 & 0 & 0 \\
E_{3} & 0 & 0 \\
0 & { }^{t} E_{3} & 0
\end{array}\right) \quad e_{10}^{b}=\left(\begin{array}{ccc}
0 & 0 & 0 \\
E_{5} & 0 & 0 \\
0 & E_{5} & 0
\end{array}\right)\right. \text {. }
$$

The representation $\rho_{2}^{b}: H_{2}^{b} \rightarrow G L(10, \mathbf{R})$ is

$$
\rho_{2}^{b}(P)=\left(\begin{array}{cccccccc}
* & 0 & 0 & 0 & 0 & * * * * & 0 & 0 \\
* & a_{1} & 0 & a_{2} & 0 & * * * & 0 & 0 \\
* & 0 & a_{1} & 0 & a_{2} & * * * & 0 & 0 \\
* & a_{2} & 0 & a_{1} & 0 & * * * & 0 & 0 \\
* & 0 & a_{2} & 0 & a_{1} & * * * & 0 & 0 \\
* & \frac{s b_{1}}{t} & -\frac{t b_{2}}{s} & -\frac{s b_{1}}{t} & -\frac{t b_{2}}{s} & * * * & 0 & 0 \\
* & \frac{s b_{1}}{t} & \frac{t b_{2}}{s} & -\frac{s b_{1}}{t} & \frac{t b_{2}}{s} & * * * & 0 & 0 \\
* & -b_{2} & b_{1} & b_{2} & b_{1} & * * * & 0 & 0 \\
* & -\left(b_{2}\right)^{2}-c & \frac{2 b_{1} b_{2}}{s} & \frac{\left(b_{2}\right)^{2}-c}{s} & \frac{2 b_{1} b_{2}}{s} & * * * \frac{1}{s} & 0 \\
* & -\frac{2 b_{1} b_{2}}{t} & \frac{\left(b_{1}\right)^{2}-c}{t} & \frac{2 b_{1} b_{2}}{t} & \frac{\left(b_{1}\right)^{2}+c}{t} & * * * & 0 & \frac{1}{t}
\end{array}\right),
$$

where $P \in H_{2}^{b}, s=a_{1}-a_{2}, t=a_{1}+a_{2}$. With respect to $\mathfrak{h}_{1}^{b}=\mathfrak{h}_{2}^{b}+\mathfrak{m}_{2}^{b}$ we have $\Omega_{1}^{b}=\Omega_{2}^{b}+\Theta_{2}^{b}$, and we set $\Theta_{2}^{b}=\sum \theta_{i}^{b} e_{i}^{b}(i=9,10)$ then 


$$
\theta_{9}^{b}=\omega_{2}^{3}-\omega_{1}^{3}, \quad \theta_{10}^{b}=\omega_{2}^{4}+\omega_{1}^{4} .
$$

By taking the exterior derivative of (3.23b), and by using (3.14), (3.15b) and the Maurer-Cartan equation (1.8), we obtain the following equations:

$$
\begin{aligned}
& d u^{*} \theta_{6}^{b}=\phi_{1} \wedge u^{*} \theta_{9}^{b}-u_{10}^{* b} \wedge \phi_{2}=0, \\
& d u^{*} \theta_{7}^{b}=-\phi_{1} \wedge u^{*} \theta_{9}^{b}-u_{10}^{* b} \wedge \phi_{2}=0, \\
& d u^{*} \theta_{8}^{b}=-\phi_{1} \wedge u^{*} \theta_{10}^{b}+u_{9}^{* b} \wedge \phi_{2}=0 .
\end{aligned}
$$

Set $u^{*} \theta_{\imath}^{b}=x_{\imath} \phi_{1}+y_{i} \phi_{2}(i=9,10)$ for some smooth functions $x_{\imath}, y_{i}$ on $M$. From (3.29b),

$$
x_{9}=y_{10}, \quad x_{10}=y_{9}=0 .
$$

We consider orbits and local sections $W_{3}^{b}$ of the action of $\left(H_{2}^{b}, \rho_{2}^{b}\right)$ on $G_{10,2}$.

$$
\rho_{2}^{b}(P)\left[\begin{array}{cc}
0 & 0 \\
1 & 0 \\
0 & 1 \\
1 & 0 \\
0 & -1 \\
0 & 0 \\
0 & 0 \\
0 & 0 \\
x_{9} & 0 \\
0 & x_{9}
\end{array}\right]=\left[\begin{array}{cc}
0 & 0 \\
t & 0 \\
0 & s \\
t & 0 \\
0 & -s \\
0 & 0 \\
0 & 0 \\
0 & 0 \\
\frac{-2 c+x_{9}}{s} & 0 \\
0 & \frac{-2 c+x_{9}}{t}
\end{array}\right]=\left[\begin{array}{cc}
0 & 0 \\
1 & 0 \\
0 & 1 \\
1 & 0 \\
0 & -1 \\
0 & 0 \\
0 & 0 \\
0 & 0 \\
\frac{-2 c+x_{9}}{\operatorname{det} A} & 0 \\
0 & \frac{-2 c+x_{9}}{\operatorname{det} A}
\end{array}\right] \text {. }
$$

Thus we take

$$
W_{3}^{b}=\left\{{ }^{t}\left[\begin{array}{llllclllll}
0 & 1 & 0 & 1 & 0 & 0 & 0 & 0 & 0 & 0 \\
0 & 0 & 1 & 0 & -1 & 0 & 0 & 0 & 0 & 0
\end{array}\right]\right\}
$$

i.e.

$$
u^{*} \theta_{9}^{b}=0, \quad u^{*} \theta_{10}^{b}=0 .
$$

Construction of fourth order frames of type (b)

The isotropy subgroup $H_{3}^{b}$ of $H_{2}^{b}$ at a point of $W_{3}^{b}$ is

$$
H_{3}^{b}=\left\{\left(\begin{array}{ccc}
A & 0 & 0 \\
0 & I_{2} & 0 \\
0 & 0 & { }^{t} A^{-1}
\end{array}\right) \exp \left(\begin{array}{ccc}
0 & 0 & 0 \\
B & 0 & 0 \\
0 & { }^{t} B & 0
\end{array}\right) ;\right.
$$




$$
\left.A=\left(\begin{array}{ll}
a_{1} & a_{2} \\
a_{2} & a_{1}
\end{array}\right) \in G L(2, \mathbf{R}), B=\left(\begin{array}{cc}
b_{1} & b_{1} \\
b_{2} & -b_{2}
\end{array}\right)\right\} .
$$

The Lie algebra $\mathfrak{h}_{3}^{b}$ of $H_{3}^{b}$ is

$$
\mathfrak{h}_{3}^{b}=\left\{\left(\begin{array}{ccc}
\alpha & 0 & 0 \\
\beta & 0 & 0 \\
0 & { }^{t} \beta & -{ }^{t} \alpha
\end{array}\right) ; \alpha=\left(\begin{array}{cc}
\alpha_{1} & \alpha_{2} \\
\alpha_{2} & \alpha_{1}
\end{array}\right), \beta=\left(\begin{array}{cc}
\beta_{1} & \beta_{1} \\
\beta_{2} & -\beta_{2}
\end{array}\right)\right\} .
$$

We decompose $\mathfrak{h}_{2}^{b}=\mathfrak{h}_{3}^{b}+\mathfrak{m}_{3}^{b}$, and for a basis of $\mathfrak{m}_{3}^{b}$ we take

$$
e_{11}^{b}=\left(\begin{array}{ccc}
0 & 0 & 0 \\
0 & 0 & 0 \\
E_{1} & 0 & 0
\end{array}\right)
$$

The representation $\rho_{3}^{b}: H_{3}^{b} \rightarrow G L(11, \mathbf{R})$ is

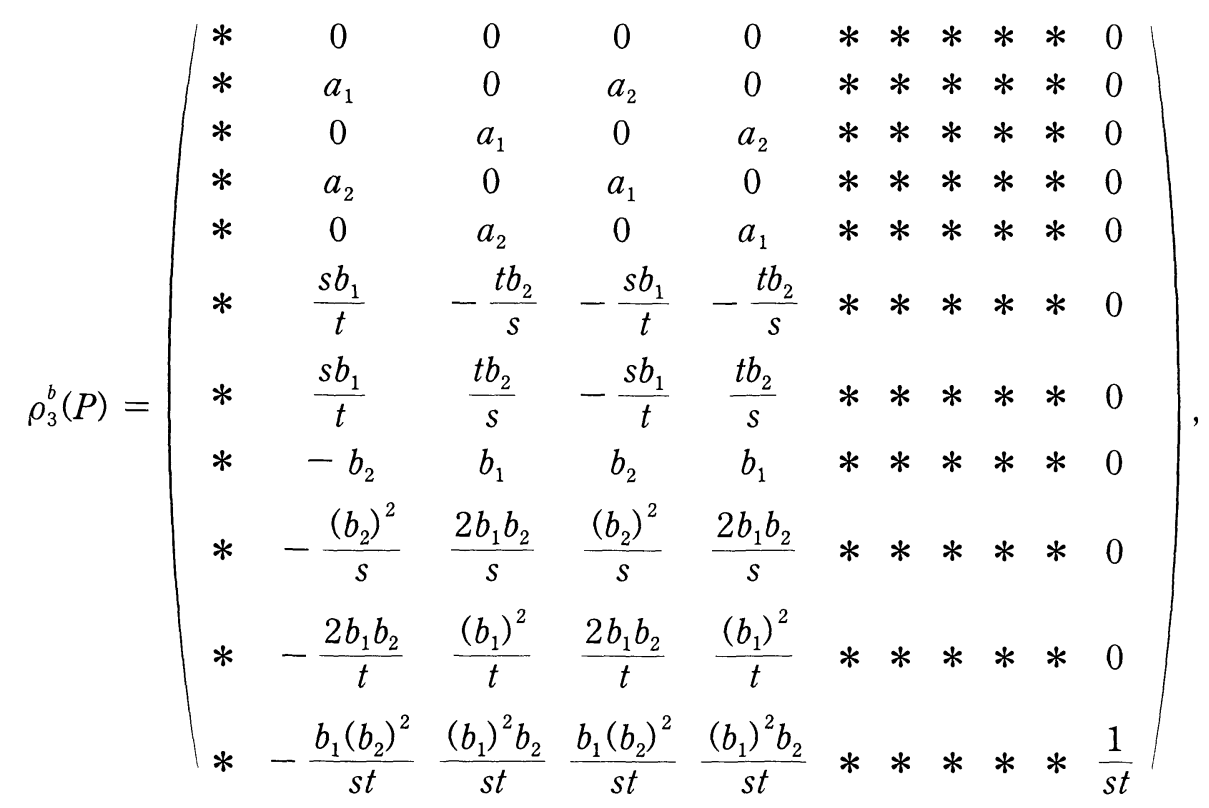

where $P \in H_{3}^{b}, s=a_{1}-a_{2}, t=a_{1}+a_{2}$. With respect to $\mathfrak{h}_{2}^{b}=\mathfrak{h}_{3}^{b}+\mathfrak{m}_{3}^{b}$ we have $\Omega_{2}^{b}=\Omega_{3}^{b}+\Theta_{3}^{b}$, and we set $\Theta_{3}^{b}=\theta_{11}^{b} e_{11}^{b}$ then

$$
\theta_{11}^{b}=\omega_{2}^{5} .
$$

By taking the exterior derivative of (3.31b), and by using (3.14), (3.15b), (3.23b) and the Maurer-Cartan equation (1.8), we obtain the following equations: 


$$
d u^{*} \theta_{9}^{b}=-2 \phi_{1} \wedge u^{*} \theta_{11}^{b}=0, \quad d u^{*} \theta_{10}^{b}=2 u^{*} \theta_{11}^{b} \wedge \phi_{2}=0 .
$$

It follows that

$$
u^{*} \theta_{11}^{b}=0
$$

Thus we take a local cross section

$$
W_{4}^{b}=\left\{{ }^{t}\left[\begin{array}{llllcllllll}
0 & 1 & 0 & 1 & 0 & 0 & 0 & 0 & 0 & 0 & 0 \\
0 & 0 & 1 & 0 & -1 & 0 & 0 & 0 & 0 & 0 & 0
\end{array}\right]\right\}
$$

of the action $\left(H_{3}^{a}, \rho_{3}^{a}\right)$ on $G_{11,2}$. For this reason, third order frame fields $u$ along $\lambda$ are fourth order frame fields along $\lambda$ with respect to $W_{4}^{b}$; i.e.

$$
u^{*}\left(\Theta_{0}+\Theta_{1}^{b}+\Theta_{2}^{b}+\Theta_{3}^{b}\right)=\phi_{1} e_{2}+\phi_{2} e_{3}+\phi_{1} e_{4}-\phi_{2} e_{5} .
$$

The isotropy subgroup $H_{4}^{b}$ of $H_{3}^{b}$ at a point of $W_{4}^{b}$ is equal to $H_{3}^{b}$. In this way we get a third order frame field $u: M \rightarrow G / H_{3}^{b}$ along $\lambda: M \rightarrow G / H$. Adding an extra step we lift $u$ from $G / H_{3}^{b}$ to $G$, then we get a Lie frame of $\lambda$. By choosing a lifting $\tilde{u}$ of $u$ such that $\tilde{u}^{*} \Omega_{3}^{b}=0$, we obtain the Lie frame of type (b) in Theorem 3.1.

\section{Type (c) and Type (d)}

Let $\lambda: M \rightarrow T_{1} S^{3}$ be of type (c); i.e. there exists a first order frame field $u$ along $\lambda$ which satisfies (3.14), (3.15b) and the following equations:

$$
u^{*}\left(\omega_{1}^{2}-\omega_{2}^{1}\right)=u^{*} \theta_{6}^{b}=\phi_{1}, u^{*}\left(\omega_{2}^{2}-\omega_{1}^{1}\right)=u^{*} \theta_{7}^{b}=\phi_{1}, u^{*} \omega_{4}^{3}=u^{*} \theta_{8}^{b}=0 .
$$

Construction of third order frames of type (b)

The isotropy subgroup $H_{2}^{c}$ of $H_{1}^{b}$ at a point of $W_{2}^{c}$ is

$$
\begin{aligned}
H_{2}^{c}=\left\{\left(\begin{array}{ccc}
A & 0 & 0 \\
0 & I_{2} & 0 \\
0 & 0 & { }^{t} A^{-1}
\end{array}\right) \exp \left(\begin{array}{ccc}
0 & 0 & 0 \\
B & 0 & 0 \\
0 & { }^{t} B & 0
\end{array}\right) \exp \left(\begin{array}{ccc}
0 & 0 & 0 \\
0 & 0 & 0 \\
C & 0 & 0
\end{array}\right) ;\right. \\
\left.A=\left(\begin{array}{ll}
a_{1} & a_{2} \\
a_{2} & a_{1}
\end{array}\right), a_{1}-a_{2}=\left(a_{1}+a_{2}\right)^{2} \neq 0, B=\left(\begin{array}{cc}
b_{1} & b_{1} \\
b_{2} & -b_{2}
\end{array}\right), C \in \mathcal{D}(2)\right\} .
\end{aligned}
$$

The Lie algebra $\mathfrak{h}_{2}^{c}$ of $H_{2}^{c}$ is

$$
\mathfrak{h}_{2}^{c}=\left\{\left(\begin{array}{ccc}
\alpha & 0 & 0 \\
\beta & 0 & 0 \\
\gamma & { }^{t} \beta & -{ }^{t} \alpha
\end{array}\right) ; \alpha=\left(\begin{array}{cc}
-3 \alpha_{2} & \alpha_{2} \\
\alpha_{2} & -3 \alpha_{2}
\end{array}\right), \beta=\left(\begin{array}{cc}
\beta_{1} & \beta_{1} \\
\beta_{2} & -\beta_{2}
\end{array}\right), \gamma \in \mathrm{o}(2)\right\} .
$$

We decompose $\mathfrak{h}_{1}^{b}=\mathfrak{h}_{2}^{c}+\mathfrak{m}_{2}^{c}$, and for a basis of $\mathfrak{m}_{2}^{c}$ we take 


$$
e_{9}^{c}=\left(\begin{array}{ccc}
0 & 0 & 0 \\
E_{3} & 0 & 0 \\
0 & { }^{t} E_{3} & 0
\end{array}\right), \quad e_{10}^{c}=\left(\begin{array}{ccc}
0 & 0 & 0 \\
E_{5} & 0 & 0 \\
0 & { }^{t} E_{5} & 0
\end{array}\right) . \quad e_{11}^{c}=\left(\begin{array}{ccc}
I_{2} & 0 & 0 \\
0 & 0 & 0 \\
0 & 0 & -{ }^{t} I_{2}
\end{array}\right)
$$

The representation $\rho_{2}^{c}: H_{2}^{c} \rightarrow G L(11, \mathbf{R})$ is

$$
(3.27 \mathrm{c})
$$

$$
\begin{aligned}
& \rho_{2}^{c}(P)= \\
& \left(\begin{array}{ccccccccccc}
* & 0 & 0 & 0 & 0 & 0 & 0 & * & 0 & 0 & 0 \\
* & a_{1} & 0 & a_{2} & 0 & 0 & 0 & * & 0 & 0 & 0 \\
* & 0 & a_{1} & 0 & a_{2} & 0 & 0 & * & 0 & 0 & 0 \\
* & a_{2} & 0 & a_{1} & 0 & 0 & 0 & * & 0 & 0 & 0 \\
* & 0 & a_{2} & 0 & a_{1} & 0 & 0 & * & 0 & 0 & 0 \\
* & t b_{1} & -\frac{b_{2}}{t} & -t b_{1} & -\frac{b_{2}}{t} & p & -q & * & 0 & 0 & 0 \\
* & t b_{1} & \frac{b_{2}}{t} & -t b_{1} & \frac{b_{2}}{t} & -q & p & * & 0 & 0 & 0 \\
* & -b_{2} & b_{1} & b_{2} & b_{1} & 0 & 0 & * & 0 & 0 & 0 \\
* & -\frac{b_{2}^{2}-c}{t^{2}} & \frac{2 b_{1} b_{2}}{t^{2}} & \frac{b_{2}^{2}-c}{t^{2}} & \frac{2 b_{1} b_{2}}{t^{2}} & -\frac{b_{1}}{t^{2}} & \frac{b_{1}}{t^{2}} & * \frac{1}{t^{2}} & 0 & 0 \\
* & -\frac{2 b_{1} b_{2}}{t} & \frac{b_{1}^{2}-c}{t} & \frac{2 b_{1} b_{2}}{t} & \frac{b_{1}^{2}+c}{t} & -\frac{b_{2}}{t} & -\frac{b_{2}}{t} & * & 0 & \frac{1}{t} & 0 \\
* & -\frac{4 a_{1} b_{1}}{t} & \frac{2 a_{1} b_{2}}{t^{2}} & -\frac{4 a_{2} b_{1}}{t} & \frac{2 a_{2} b_{2}}{t^{2}} & \frac{a_{1} a_{2}-3 a_{2}^{2}}{t^{3}} & \frac{-a_{2}^{2}+3 a_{1} a_{2}}{t^{3}} & * & 0 & 0 & 1
\end{array}\right) \text {, }
\end{aligned}
$$

where $P \in H_{2}^{c}, t=a_{1}+a_{2}, p=\frac{a_{1}^{2}+a_{2}^{2}}{t^{3}}, q=\frac{2 a_{1} a_{2}}{t^{3}}$. With respect to $\mathfrak{h}_{1}^{b}=\mathfrak{h}_{2}^{c}+$ $\mathrm{m}_{2}^{c}$ we have $\Omega_{1}^{b}=\Omega_{2}^{c}+\Theta_{2}^{c}$, and we set $\Theta_{2}^{c}=\sum \theta_{i}^{c} e_{\imath}^{c}(i=9,10,11)$ then

$$
\theta_{9}^{c}=\omega_{2}^{3}-\omega_{1}^{3}, \theta_{10}^{c}=\omega_{2}^{4}+\omega_{1}^{4}, \theta_{11}^{b}=\omega_{1}^{1}+3 \omega_{2}^{1} .
$$

By taking the exterior derivative of (3.23c), and by using (3.14), (3.15b) and the Maurer-Cartan equation (1.8), we obtain the following equations:

$$
\begin{aligned}
& d\left(u^{*} \theta_{6}^{b}-\phi_{1}\right)=\phi_{1} \wedge u^{*} \theta_{9}^{c}-u^{*} \theta_{10}^{c} \wedge \phi_{2}-\phi_{1} \wedge u^{*} \theta_{11}^{c}=0, \\
& d\left(u^{*} \theta_{7}^{b}-\phi_{2}\right)=-\phi_{1} \wedge u^{*} \theta_{9}^{c}-u^{*} \theta_{10}^{c} \wedge \phi_{2}-\phi_{1} \wedge u^{*} \theta_{11}^{c}=0, \\
& d u^{*} \theta_{8}^{b}=u^{*} \theta_{9}^{c} \wedge \phi_{2}-\phi_{1} \wedge u^{*} \theta_{10}^{c}=0 .
\end{aligned}
$$

Set $u^{*} \theta_{i}^{c}=x_{i} \phi_{1}+y_{i} \phi_{2}(i=9,10,11)$ for some smooth functions $x_{i}, y_{\imath}$ on $M$. From (3.29c), 


$$
x_{9}=y_{10}, \quad x_{10}=-y_{11}, \quad y_{9}=0 .
$$

We consider orbits and local sections $W_{3}^{c}$ of the action of $\left(H_{2}^{c}, \rho_{2}^{c}\right)$ on $G_{11,2}$.

$$
\rho_{2}^{c}(P)\left[\begin{array}{cc}
0 & 0 \\
1 & 0 \\
0 & 1 \\
1 & 0 \\
0 & -1 \\
1 & 0 \\
1 & 0 \\
0 & 0 \\
x_{9} & 0 \\
x_{10} & x_{9} \\
x_{11} & -x_{10}
\end{array}\right]=\left[\begin{array}{cc}
0 & 0 \\
t & 0 \\
0 & t^{2} \\
t & 0 \\
0 & -t^{2} \\
t & 0 \\
t & 0 \\
0 & 0 \\
\frac{-2 c+x_{9}}{t^{2}} & 0 \\
\frac{-2 b_{2}+x_{10}}{t} & \frac{-2 c+x_{9}}{t} \\
\frac{4\left(a_{2}-t b_{1}\right)+t x_{11}}{t} & 2 b_{2}-x_{10}
\end{array}\right]=\left[\begin{array}{ccc}
0 & 0 \\
1 & 0 \\
0 & 1 \\
1 & 0 \\
0 & -1 \\
1 & 0 \\
1 & 0 \\
0 & 0 \\
\frac{-2 c+x_{9}}{t^{3}} & 0 \\
\frac{-2 b_{2}+x_{10}}{t^{2}} & \frac{-2 c+x_{9}}{t^{3}} \\
\frac{4\left(a_{2}-t b_{1}\right)+t x_{11}}{t^{2}} & \frac{2 b_{2}-x_{10}}{t^{2}}
\end{array}\right] .
$$

Thus we take

$$
W_{3}^{c}=\left\{{ }^{t}\left[\begin{array}{ccccccccccc}
0 & 1 & 0 & 1 & 0 & 1 & 1 & 0 & 0 & 0 & 0 \\
0 & 0 & 1 & 0 & -1 & 0 & 0 & 0 & 0 & 0 & 0
\end{array}\right]\right\}
$$

i.e.

$$
u^{*} \theta_{9}^{c}=0, \quad u^{*} \theta_{10}^{c}=0, \quad u^{*} \theta_{11}^{c}=0 .
$$

Construction of fourth order frames of type (c)

The isotropy subgroup $H_{3}^{c}$ of $H_{2}^{c}$ at a point of $W_{3}^{c}$ is

$$
\begin{aligned}
& H_{3}^{c}=\left(\begin{array}{ccc}
A & 0 & 0 \\
0 & I_{2} & 0 \\
0 & 0 & { }^{t} A^{-1}
\end{array}\right) \exp \left(\begin{array}{ccc}
0 & 0 & 0 \\
B & 0 & 0 \\
0 & { }^{t} B & 0
\end{array}\right) ; A=\left(\begin{array}{ll}
a_{1} & a_{2} \\
a_{2} & a_{1}
\end{array}\right), \\
&\left.a_{1}-a_{2}=\left(a_{1}+a_{2}\right)^{2} \neq 0, B=\left(\begin{array}{cc}
b_{1} & b_{1} \\
0 & 0
\end{array}\right), b_{1}=\frac{a_{2}}{a_{1}+a_{2}}\right\} .
\end{aligned}
$$

The Lie algebra $\mathfrak{h}_{3}^{c}$ of $H_{3}^{c}$ is

(3.33c) $\mathfrak{h}_{3}^{c}=\left\{\left(\begin{array}{ccc}\alpha & 0 & 0 \\ \beta & 0 & 0 \\ 0 & { }^{t} \beta & -{ }^{t} \alpha\end{array}\right) ; \alpha=\left(\begin{array}{cc}-3 \alpha_{2} & \alpha_{2} \\ \alpha_{2} & -3 \alpha_{2}\end{array}\right), \beta=\left(\begin{array}{cc}\alpha_{2} & \alpha_{2} \\ 0 & 0\end{array}\right)\right\}$. 
We decompose $\mathfrak{h}_{2}^{c}=\mathfrak{h}_{3}^{c}+\mathfrak{m}_{3}^{c}$, and for a basis of $\mathfrak{m}_{3}^{c}$ we take

$$
\begin{aligned}
& e_{12}^{c}=\left(\begin{array}{cccccc}
0 & 0 & 0 & 0 & 0 & 0 \\
0 & 0 & 0 & 0 & 0 & 0 \\
1 & 1 & 0 & 0 & 0 & 0 \\
0 & 0 & 0 & 0 & 0 & 0 \\
0 & 0 & 1 & 0 & 0 & 0 \\
0 & 0 & 1 & 0 & 0 & 0
\end{array}\right), e_{13}^{c}=\left(\begin{array}{cccccc}
0 & 0 & 0 & 0 & 0 & 0 \\
0 & 0 & 0 & 0 & 0 & 0 \\
0 & 0 & 0 & 0 & 0 & 0 \\
1 & -1 & 0 & 0 & 0 & 0 \\
0 & 0 & 0 & 1 & 0 & 0 \\
0 & 0 & 0 & -1 & 0 & 0
\end{array}\right), \\
& e_{14}^{c}=\left(\begin{array}{lll}
0 & 0 & 0 \\
0 & 0 & 0 \\
E_{1} & 0 & 0
\end{array}\right) .
\end{aligned}
$$

The representation $\rho_{3}^{\mathrm{c}}: H_{3}^{c} \rightarrow G L(13, \mathbf{R})$ is

$$
\begin{aligned}
& \rho_{3}^{c}(P)=\left(\begin{array}{ll}
\bar{\rho}_{1}^{1} & \bar{\rho}_{2}^{1} \\
\bar{\rho}_{1}^{2} & \bar{\rho}_{2}^{2}
\end{array}\right),
\end{aligned}
$$

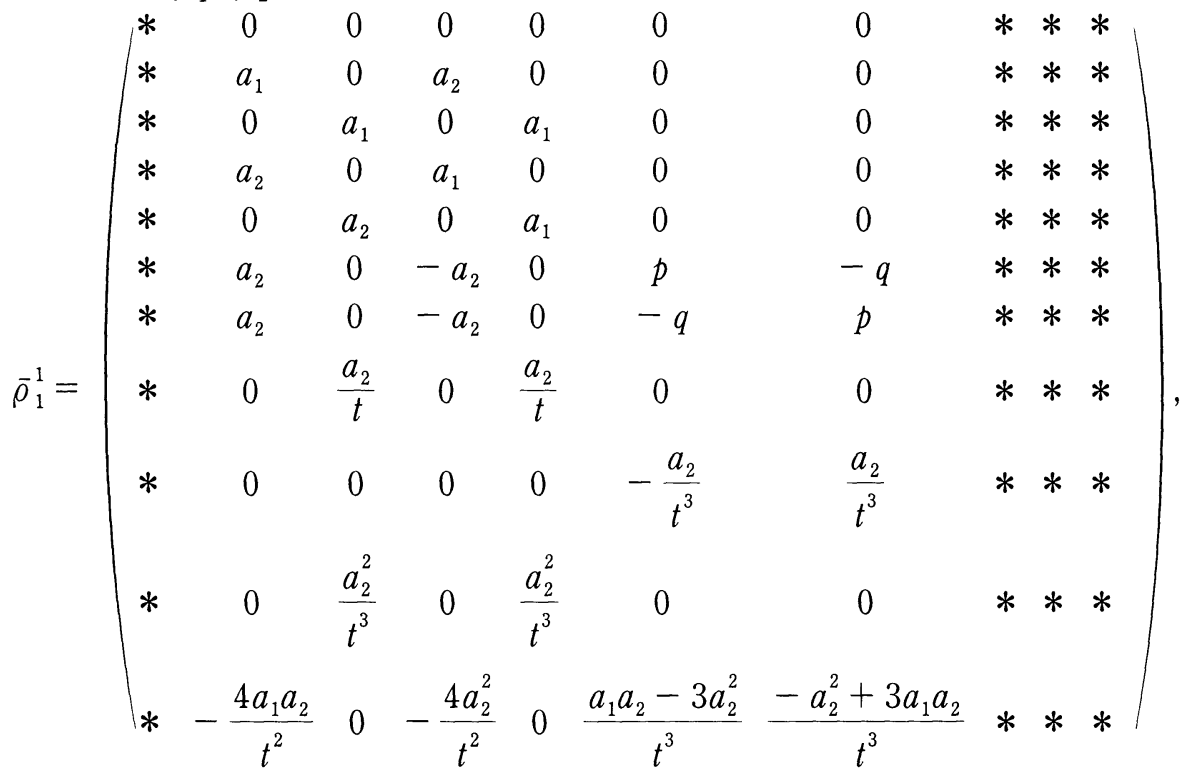

$$
\begin{aligned}
& \bar{\rho}_{2}^{1}=0 \in M_{10,3} \text {, }
\end{aligned}
$$

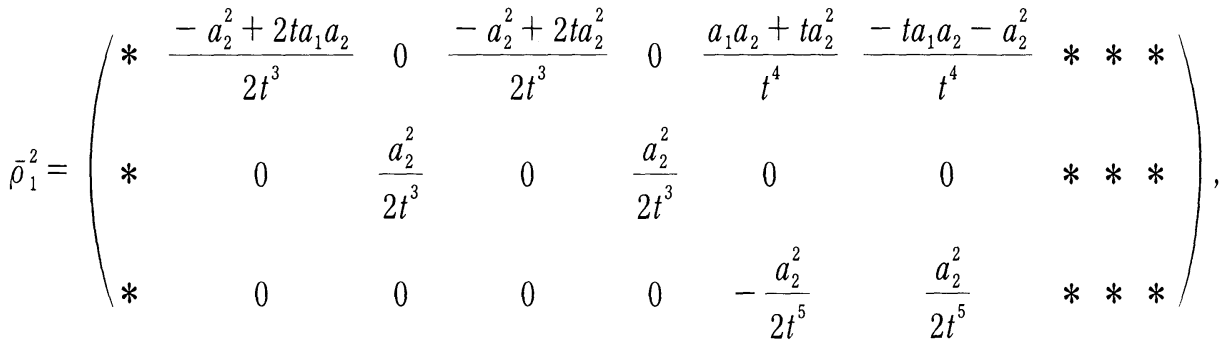


$\bar{\rho}_{2}^{2}=\left(\begin{array}{ccc}\frac{1}{t} & 0 & 0 \\ 0 & \frac{1}{t^{2}} & 0 \\ 0 & 0 & \frac{1}{t^{3}}\end{array}\right)$,

where $P \in H_{3}^{c}, t=a_{1}+a_{2}, p=\frac{a_{1}^{2}+a_{2}^{2}}{t^{3}}, q=\frac{2 a_{1} a_{2}}{t^{3}}$. With respect to $\mathfrak{h}_{2}^{c}=\mathfrak{h}_{3}^{c}$.

$\mathrm{m}_{3}^{c}$ we have $\Omega_{2}^{c}=\Omega_{3}^{c}+\Theta_{3}^{c}$, and we set $\Theta_{3}^{c}=\sum \theta_{i}^{c} e_{\imath}^{c}(i=12,13,14)$ then

$$
\theta_{12}^{c}=\omega_{1}^{3}-\omega_{2}^{1}, \quad \theta_{13}^{c}=\omega_{1}^{4}, \quad \theta_{14}^{c}=\omega_{2}^{5} .
$$

By taking the exterior derivative of (3.31c), and by using (3.14), (3.15b), (3.23c) and the Maurer-Cartan equation (1.8), we obtain the following equations:

$$
\begin{aligned}
& d u^{*} \theta_{9}^{c}=-2 \phi_{1} \wedge u^{*} \theta_{14}^{c}=0, \\
& d u^{*} \theta_{10}^{c}=-2 \phi_{1} \wedge u^{*} \theta_{13}^{c}+2 u^{*} \theta_{14}^{c} \wedge \phi_{2}=0, \\
& d u^{*} \theta_{11}^{c}=-4 \phi_{1} \wedge u^{*} \theta_{12}^{c}-2 u^{*} \theta_{13}^{c} \wedge \phi_{2}=0 .
\end{aligned}
$$

Set $u^{*} \theta_{\imath}^{c}=x_{\imath} \phi_{1}+y_{\imath} \phi_{2}(i=12,13,14)$ for some smooth functions $x_{\imath}, y_{\imath}$ on $M$. From $(3.37 \mathrm{c})$,

$$
x_{13}=-2 y_{12}=2 k^{3}, \quad x_{14}=y_{13}=k^{4}, \quad y_{14}=0 .
$$

We consider orbits and local sections $W_{4}^{c}$ of the action of $\left(H_{3}^{c}, \rho_{3}^{c}\right)$ on $G_{13,2}$.

$$
\rho_{3}^{c}(P)\left[\begin{array}{cc}
0 & 0 \\
1 & 0 \\
0 & 1 \\
1 & 0 \\
0 & -1 \\
1 & 0 \\
1 & 0 \\
0 & 0 \\
0 & 0 \\
0 & 0 \\
0 & 0 \\
x_{12} & -k^{3} \\
2 k^{3} & k^{4} \\
k^{4} & 0
\end{array}\right]=\left[\begin{array}{cc}
0 & 0 \\
t & 0 \\
0 & t^{2} \\
t & 0 \\
0 & -t^{2} \\
t & 0 \\
t & 0 \\
0 & 0 \\
0 & 0 \\
0 & 0 \\
0 & 0 \\
\frac{a_{1} a_{2}}{t^{3}}+\frac{1}{t} x_{12} & -\frac{1}{t} k^{3} \\
\frac{2}{t^{2}} k^{3} & \frac{1}{t^{2}} k^{4} \\
\frac{1}{t^{3}} k^{4} & 0
\end{array}\right]=\left[\begin{array}{cc}
0 & 0 \\
1 & 0 \\
0 & 1 \\
1 & 0 \\
0 & -1 \\
1 & 0 \\
1 & 0 \\
0 & 0 \\
0 & 0 \\
0 & 0 \\
0 & 0 \\
\frac{a_{1} a_{2}}{t^{4}}+\frac{1}{t^{2}} x_{12} & -\frac{1}{t^{3}} k^{3} \\
\frac{2}{t^{3}} k^{3} & \frac{1}{t^{4}} k^{4} \\
\frac{1}{t^{4}} k^{4} & 0
\end{array}\right]
$$


Thus we take

$$
W_{4}^{c}=\left\{{ }^{t}\left[\begin{array}{cccccccccccccc}
0 & 1 & 0 & 1 & 0 & 1 & 1 & 0 & 0 & 0 & 0 & 0 & 2 k^{3} & k^{4} \\
0 & 0 & 1 & 0 & -1 & 0 & 0 & 0 & 0 & 0 & 0 & -k^{3} & k^{4} & 0
\end{array}\right]\right\}
$$

i.e

$$
u^{*} \theta_{12}^{c}=-k^{3} \phi_{2}, \quad u^{*} \theta_{13}^{c}=2 k^{3} \phi_{1}+k^{4} \phi_{2}, \quad u^{*} \theta_{14}^{c}=k^{4} \phi_{1} .
$$

Construction of fifth order frames of type (c)

The isotropy subgroup $H_{4}^{c}$ of $H_{3}^{c}$ is equal to $\left\{I_{6}\right\}$. For a basis of $\mathfrak{h}_{3}^{c}$ we take

$$
e_{15}^{c}=\left(\begin{array}{cccccc}
-3 & 1 & 0 & 0 & 0 & 0 \\
1 & -3 & 0 & 0 & 0 & 0 \\
1 & 1 & 0 & 0 & 0 & 0 \\
0 & 0 & 0 & 0 & 0 & 0 \\
0 & 0 & 1 & 0 & 3 & -1 \\
0 & 0 & 1 & 0 & -1 & 3
\end{array}\right)
$$

We set $\Omega_{3}^{c}=\theta_{15}^{c} e_{15}^{c}$ then

$$
\theta_{15}^{c}=\omega_{2}^{1}
$$

Set

$$
\begin{aligned}
& u^{*} \theta_{15}^{c}=k^{1} \phi_{1}+k^{2} \phi_{2}, \\
& d k^{i}=k_{1}^{i} \phi_{1}+k_{2}^{i} \phi_{2}(i=1,2,3,4),
\end{aligned}
$$

for some smooth functions $k^{\imath}, k_{\text {, }}^{\imath}$ on $M$. By taking the exterior derivative of (3.40c), and by using (3.14), (3.15b), (3.23c), (3.31c), (3.44c) and the Maurer-Cartan equation (1.8), we obtain the following equations:

$$
\begin{aligned}
& d\left(u^{*} \theta_{12}^{c}+k^{3} \phi_{2}\right)=\phi_{1} \wedge u^{*} \theta_{15}^{c}+6 k^{3} u^{*} \theta_{15}^{c} \wedge \phi_{2}+k_{1}^{3} \phi_{1} \wedge \phi_{2}=0 \\
& d\left(u^{*} \theta_{13}^{c}-\left(2 k^{3} \phi_{1}+k^{4} \phi_{2}\right)\right) \\
& \quad=12 k^{3} \phi_{1} \wedge u^{*} \theta_{15}^{c}-8 k^{4} u^{*} \theta_{15}^{c} \wedge \phi_{2}+\left(2 k_{2}^{3}-k_{1}^{4}\right) \phi_{1} \wedge \phi_{2}=0 \\
& d\left(u^{*} \theta_{14}^{c}-k^{4} \phi_{1}\right)=8 k^{4} \phi_{1} \wedge u^{*} \theta_{15}^{c}+k_{2}^{4} \phi_{1} \wedge \phi_{2}=0 .
\end{aligned}
$$

From (3.43c), (3.44c), and (3.45c),

$$
\begin{aligned}
& k^{2}+6 k^{1} k^{3}+k_{1}^{3}=0, \\
& 12 k^{2} k^{3}-8 k^{1} k^{4}+\left(2 k_{2}^{3}-k_{1}^{4}\right)=0, \\
& 8 k^{2} k^{4}+k_{2}^{4}=0 .
\end{aligned}
$$

Thus we take a local cross section 


$$
W_{5}^{c}=\left\{\left\{^{t}\left[\begin{array}{ccccccccccccccc}
0 & 1 & 0 & 1 & 0 & 1 & 1 & 0 & 0 & 0 & 0 & 0 & 2 k^{3} & k^{4} & k^{1} \\
0 & 0 & 1 & 0 & -1 & 0 & 0 & 0 & 0 & 0 & 0 & -k^{3} & k^{4} & 0 & k^{2}
\end{array}\right]\right\}\right.
$$

of the trivial adjoint action $\left(H_{4}^{c}, \rho_{4}^{c}\right)$ on $G_{15,2}$, where $k^{i}$ are smooth functions on $M$ that satisfy the conditions $(3.46 \mathrm{c})$. By taking the exterior derivative (3.43c), and by using (3.14), (3.15b), (3.23c), (3.31c), (3.40c) and the Maurer-Cartan equation (1.8), we obtain the following equations:

$$
d\left(u^{*} \theta_{15}^{c}-\left(k^{1} \phi_{1}+k^{2} \phi_{2}\right)\right)=\left(-2 k^{1} k^{2}+k^{3}+k_{2}^{1}-k_{1}^{2}\right) \phi_{1} \wedge \phi_{2}=0 .
$$

Then

$$
2 k^{1} k^{2}-k^{3}-k_{2}^{1}+k_{1}^{2}=0 .
$$

Thus the Lie frame of a Legendre map $\lambda: M \rightarrow T_{1} S^{3}$ of type (c) is the fifth order frame field $u$ along $\lambda$ with respect to $W_{5}^{c}$. In this way we obtain the Lie frame of type (c) in Theorem 3.1 .

In precisely the same fashion as the case of type (c), we obtain the Lie frame of type (d) in Theorem 3.1.

\section{Type (e)}

Let $\lambda: M \rightarrow T_{1} S^{3}$ is of type (e); i.e. there exists order frame field $u$ along $\lambda$ which satiafies (3.14), (3.15b) and the following equations:

$$
\begin{aligned}
& u^{*}\left(\omega_{1}^{2}-\omega_{2}^{1}\right)=u^{*} \theta_{6}^{b}=\phi_{1}+\phi_{2}, u^{*}\left(\omega_{2}^{2}-\omega_{1}^{1}\right)=u^{*} \theta_{7}^{b}=\phi_{1}-\phi_{2}, \\
& u^{*} \omega_{4}^{3}=u^{*} \theta_{8}^{b}=0 .
\end{aligned}
$$

Construction of third order frames of type (e)

The isotropy subgroup $H_{2}^{e}$ of $H_{1}^{b}$ at a point of $W_{2}^{e}$ is

$$
H_{2}^{e}=\left\{\exp \left(\begin{array}{ccc}
0 & 0 & 0 \\
B & 0 & 0 \\
0 & { }^{t} B & 0
\end{array}\right) \exp \left(\begin{array}{ccc}
0 & 0 & 0 \\
0 & 0 & 0 \\
C & 0 & 0
\end{array}\right) ; B=\left(\begin{array}{cc}
b_{1} & b_{1} \\
b_{2} & -b_{2}
\end{array}\right), C \in \mathfrak{o}(2)\right\}
$$

The Lie algebra $\mathfrak{h}_{2}^{e}$ of $H_{2}^{e}$ is

$$
\mathfrak{h}_{2}^{e}=\left\{\left(\begin{array}{ccc}
0 & 0 & 0 \\
\beta & 0 & 0 \\
\gamma & { }^{t} \beta & 0
\end{array}\right) ; \beta=\left(\begin{array}{cc}
\beta_{1} & \beta_{1} \\
\beta_{2} & -\beta_{2}
\end{array}\right), \gamma \in \mathfrak{o}(2)\right\} .
$$

We decompose $\mathfrak{h}_{1}^{b}=\mathfrak{h}_{2}^{e}+\mathfrak{m}_{2}^{e}$, and for a basis of $\mathfrak{m}_{2}^{e}$ we take 
$(3.26 \mathrm{e})$

$$
\begin{aligned}
& e_{9}^{e}=\left(\begin{array}{ccc}
0 & 0 & 0 \\
E_{3} & 0 & 0 \\
0 & { }^{t} E_{3} & 0
\end{array}\right), \quad e_{10}^{e}=\left(\begin{array}{ccc}
0 & 0 & 0 \\
E_{5} & 0 & 0 \\
0 & { }^{t} E_{5} & 0
\end{array}\right) \\
& e_{11}^{e}=\left(\begin{array}{ccc}
I_{2} & 0 & 0 \\
0 & 0 & 0 \\
0 & 0 & -{ }^{t} I_{2}
\end{array}\right) \cdot e_{12}^{e}=\left(\begin{array}{cccccc}
0 & 1 & 0 & 0 & 0 & 0 \\
1 & 0 & 0 & 0 & 0 & 0 \\
0 & 0 & 0 & 0 & 0 & 0 \\
0 & 0 & 0 & 0 & 0 & 0 \\
0 & 0 & 0 & 0 & 0 & -1 \\
0 & 0 & 0 & 0 & -1 & 0
\end{array}\right) \text {. }
\end{aligned}
$$

The representation $\rho_{2}^{e}: H_{2}^{e} \rightarrow G L(15, \mathbf{R})$ is

(3.27e)

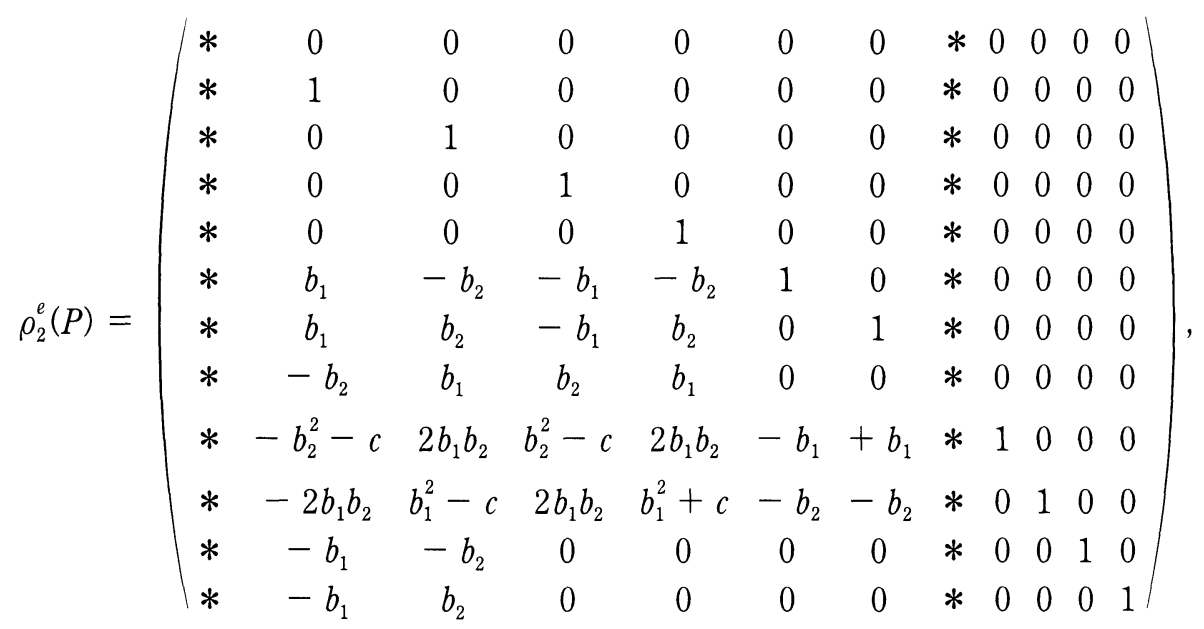

where $P \in H_{2}^{e}$. With respect to $\mathfrak{h}_{1}^{b}=\mathfrak{h}_{2}^{e}+\mathfrak{m}_{2}^{e}$ we have $\Omega_{1}^{b}=\Omega_{2}^{e}+\Theta_{2}^{e}$, and we set $\Theta_{2}^{e}=\sum \theta_{i}^{e} e_{i}^{e}(i=9,10,11,12)$ then

$$
\theta_{9}^{e}=\omega_{2}^{3}-\omega_{1}^{3}, \theta_{10}^{e}=\omega_{2}^{4}+\omega_{1}^{4}, \theta_{11}^{e}=\omega_{1}^{1}, \theta_{12}^{e}=\omega_{2}^{1} .
$$

By taking the exterior derivative of (3.23e), and by using (3.14), (3.15b) and the Maurer-Cartan equation (1.8), we obtain the following equations:

$$
\begin{aligned}
d\left(u^{*} \theta_{6}^{b}\right. & \left.-\left(\phi_{1}+\phi_{2}\right)\right) \\
= & \phi_{1} \wedge\left(u^{*} \theta_{9}^{e}-u^{*} \theta_{11}^{e}-3 u^{*} \theta_{12}^{e}\right)+\left(-u^{*} \theta_{10}^{e}+u^{*} \theta_{11}^{e}-3 u^{*} \theta_{12}^{e}\right) \wedge \phi_{2} \\
& -2 \phi_{1} \wedge \phi_{2}=0, \\
(3.29 \mathrm{e}) \quad d\left(u^{*} \theta_{7}^{b}-\left(\phi_{1}+\phi_{2}\right)\right) & \\
= & \phi_{1} \wedge\left(-u^{*} \theta_{9}^{e}-u^{*} \theta_{11}^{e}-3 u^{*} \theta_{12}^{e}\right)+\left(-u^{*} \theta_{10}^{e}-u^{*} \theta_{11}^{e}+3 u^{*} \theta_{12}^{e}\right)
\end{aligned}
$$




$$
\begin{gathered}
\wedge \phi_{2}=0, \\
d u^{*} \theta_{8}^{b}=-\phi_{1} \wedge u^{*} \theta_{10}^{e}+u^{*} \theta_{9}^{e} \wedge \phi_{2}=0 .
\end{gathered}
$$

Set $u^{*} \theta_{i}^{e}=x_{\imath} \phi_{1}+y_{\imath} \phi_{2}(i=9,10,11,12)$ for some smooth functions $x_{\imath}, y_{\imath}$ on $M$. From (3.29e),

$$
x_{10}+y_{11}+3 y_{12}+1=0, \quad y_{9}+x_{11}-3 x_{12}-1=0, \quad x_{9}=y_{10} .
$$

We consider orbits and local sections $W_{3}^{e}$ of the action of $\left(H_{2}^{e}, \rho_{2}^{e}\right)$ on $G_{12,2}$.

Thus we take

$$
\rho_{2}^{e}(P)\left[\begin{array}{cc}
0 & 0 \\
1 & 0 \\
0 & 1 \\
1 & 0 \\
0 & -1 \\
1 & 1 \\
1 & -1 \\
0 & 0 \\
x_{9} & y_{9} \\
x_{10} & y_{10} \\
x_{11} & y_{11} \\
x_{12} & x_{12}
\end{array}\right]=\left[\begin{array}{cc}
0 & 0 \\
1 & 0 \\
0 & 1 \\
1 & 0 \\
0 & -1 \\
1 & 1 \\
1 & -1 \\
0 & 0 \\
-2 c+x_{9} & -2 b_{1}+y_{9} \\
-2 b_{2}+x_{10} & -2 c+y_{10} \\
-b_{1}+x_{11} & -b_{2}+y_{11} \\
-b_{1}+x_{12} & b_{2}+x_{12}
\end{array}\right] .
$$

$$
W_{3}^{e}=\left\{{ }^{t}\left[\begin{array}{cccccccccccc}
0 & 1 & 0 & 1 & 0 & 1 & 1 & 0 & 0 & 0 & 3 k^{1}+1 & k^{1} \\
0 & 0 & 1 & 0 & -1 & 1 & -1 & 0 & 0 & 0 & -3 k^{2}-1 & k^{2}
\end{array}\right]\right\}
$$
i.e

$$
\begin{aligned}
& u^{*} \theta_{9}^{e}=0, \quad u^{*} \theta_{10}^{e}=0, \\
& u^{*} \theta_{11}^{e}=\left(3 k^{1}+1\right) \phi_{1}+\left(-3 k^{3}-1\right) \phi_{2}, \quad u^{*} \theta_{12}^{e}=k^{1} \phi_{1}+k^{2} \phi_{2} .
\end{aligned}
$$

Construction of fourth order frames of type (e)

The isotropy subgroup $H_{3}^{e}$ of $H_{2}^{e}$ is equal to $\left\{I_{6}\right\}$. For a basis of $\mathfrak{h}_{2}^{e}$ we take

$$
\begin{aligned}
& e_{13}^{e}=\left(\begin{array}{llllll}
0 & 0 & 0 & 0 & 0 & 0 \\
0 & 0 & 0 & 0 & 0 & 0 \\
1 & 1 & 0 & 0 & 0 & 0 \\
0 & 0 & 0 & 0 & 0 & 0 \\
0 & 0 & 1 & 0 & 0 & 0 \\
0 & 0 & 1 & 0 & 0 & 0
\end{array}\right), e_{14}^{e}=\left(\begin{array}{cccccc}
0 & 0 & 0 & 0 & 0 & 0 \\
0 & 0 & 0 & 0 & 0 & 0 \\
0 & 0 & 0 & 0 & 0 & 0 \\
1 & -1 & 0 & 0 & 0 & 0 \\
0 & 0 & 0 & 1 & 0 & 0 \\
0 & 0 & 0 & -1 & 0 & 0
\end{array}\right), \\
& e_{15}^{e}=\left(\begin{array}{lll}
0 & 0 & 0 \\
0 & 0 & 0 \\
E_{1} & 0 & 0
\end{array}\right)
\end{aligned}
$$


We set $\Omega_{2}^{c}=\sum \theta_{\imath}^{e} e_{\imath}^{e}(i=13,14,15)$ then

$$
\theta_{13}^{e}=\omega_{1}^{3}, \quad \theta_{14}^{e}=\omega_{1}^{4}, \quad \theta_{15}^{e}=\omega_{2}^{5} .
$$

Set

$$
d k^{\imath}=k_{1}^{\imath} \phi_{1}+k_{2}^{\imath} \phi_{2}(i=1,2) .
$$

By taking the exterior derivative of (3.31e), and by using (3.14), (3.15b), (3.23e), (3.34e) and the Maurer-Cartan equation (1.8), we obtain the following equations:

$$
\begin{aligned}
& d u^{*} \theta_{9}^{e}=-2 \phi_{1} \wedge u^{*} \theta_{15}^{e}+2 u^{*} \theta_{13}^{e} \wedge \phi_{2}=0 \\
& d u^{*} \theta_{10}^{e}=-2 \phi_{1} \wedge u^{*} \theta_{14}^{e}+2 u^{*} \theta_{15}^{e} \wedge \phi_{2}=0 \\
& d\left(u^{*} \theta_{11}^{b}-\left(\left(3 k^{1}+1\right) \phi_{1}+\left(-3 k^{2}-1\right) \phi_{2}\right)\right) \\
& \quad=-\phi_{1} \wedge u^{*} \theta_{13}^{e}+u^{*} \theta_{14}^{e} \wedge \phi_{2}+\left(3 k_{2}^{1}+3 k_{1}^{2}\right) \phi_{1} \wedge \phi_{2}=0 \\
& d\left(u^{*} \theta_{12}^{e}-\left(k^{1} \phi_{1}+k^{2} \phi_{2}\right)\right) \\
& \quad=-\phi_{1} \wedge u^{*} \theta_{13}^{e}-u^{*} \theta_{14}^{e} \wedge \phi_{2}+\left(k_{2}^{1}-k_{1}^{2}+2 k^{1}+2 k^{2}+4 k^{1} k^{2}\right) \phi_{1} \wedge \phi_{2} \\
& \quad=0
\end{aligned}
$$

Set $u^{*} \theta_{i}^{e}=x_{\imath} \phi_{1}+y_{i} \phi_{2}(i=13,14,15)$ for some smooth functions $x_{\imath}, y_{\imath}$ on $M$. From (3.35e),

$$
\begin{aligned}
& x_{13}=y_{15}=k^{5}, \quad x_{15}=y_{14}=k^{6}, \\
& x_{13}=\left(k^{1}+k^{2}\right)^{2}+2 k_{2}^{1}+k_{1}^{2}=k^{3}, \quad x_{14}=\left(k^{1}+k^{2}\right)^{2}-k_{2}^{1}+2 k_{1}^{2}=k^{4} .
\end{aligned}
$$

Thus we take a local cross section

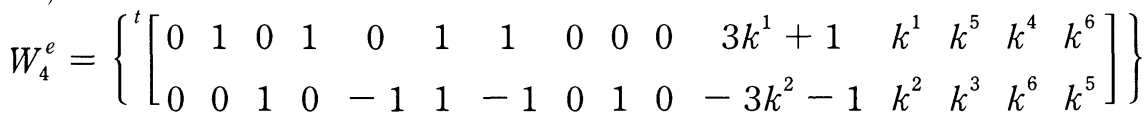

of the trivial adjoint action $\left(H_{3}^{e}, \rho_{3}^{e}\right)$ on $G_{15,2}$; i.e.

$$
u^{*} \theta_{13}^{e}=k^{5} \phi_{1}+k^{3} \phi_{2}, \quad u^{*} \theta_{14}^{e}=k^{4} \phi_{1}+k^{6} \phi_{2}, \quad u^{*} \theta_{15}^{e}=k^{6} \phi_{1}+k^{5} \phi_{2} .
$$

By taking the exterior derivative of (3.38e), and by using (3.14), (3.15b), (3.23e), (3.34e) and the Maurer-Cartan equation (1.8), we obtain the following equations:

$$
\begin{gathered}
d u^{*}\left(\theta_{13}^{e}-\left(k^{5} \phi_{1}+k^{3} \phi_{2}\right)\right) \\
=\left(6 k^{1} k^{3}+4 k^{2} k^{5}+2 k^{5}+3 k^{3}+k_{2}^{5}-k_{1}^{3}\right) \phi_{1} \wedge \phi_{2}=0, \\
(3.39 \mathrm{e}) d u^{*}\left(\theta_{14}^{e}-\left(k^{4} \phi_{1}+k^{6} \phi_{2}\right)\right) \\
=\left(4 k^{1} k^{6}+6 k^{2} k^{4}+2 k^{6}+3 k^{4}+k_{2}^{4}-k_{1}^{6}\right) \phi_{1} \wedge \phi_{2}=0,
\end{gathered}
$$




$$
\begin{aligned}
& d\left(u^{*} \theta_{15}^{e}-\left(k^{6} \phi_{1}+k^{5} \phi_{2}\right)\right) \\
& \quad=\left(-4 k^{2} k^{6}-4 k^{1} k^{5}-2 k^{5}-2 k^{6}+k_{1}^{5}+k_{2}^{6}\right) \phi_{1} \wedge \phi_{2}=0 .
\end{aligned}
$$

Then

$$
\begin{aligned}
& 6 k^{1} k^{3}+4 k^{2} k^{5}+2 k^{5}+3 k^{3}+k_{2}^{5}-k_{1}^{3}=0, \\
& 4 k^{1} k^{6}+6 k^{2} k^{4}+2 k^{6}+3 k^{4}+k_{2}^{4}-k_{1}^{6}=0, \\
& -4 k^{2} k^{6}-4 k^{1} k^{5}-2 k^{5}-2 k^{6}-k_{1}^{5}+k_{2}^{6}=0 .
\end{aligned}
$$

Thus the Lie frame of a Legendre map $\lambda: M \rightarrow T_{1} S^{3}$ of typy (e) is the fourth order frame field $u$ along $\lambda$ with respsct to $W_{4}^{e}$. In this way we obtain the Lie frame of type (e) in Theorem 3.1.

We have thus proved Theorem 3.1.

\section{Classification of Legendre maps in $T_{1} S^{3}$ in view of curvature spheres}

Let us consider the classification of surfaces obtained in Theorem 3.1 in view of curvature spheres. The curvature sphere of $\lambda: M^{2} \rightarrow T_{1} S^{3}$ of type (a) is $[K]=$ $\left[Y_{6}\right]$, which has multiplicity 2 . The curvature spheres of $\lambda$ of type (b), (c), (d) and (e) are

$$
\left[K_{1}(p)\right]=\left[Y_{5}(p)+Y_{6}(p)\right], \quad\left[K_{2}(p)\right]=\left[-Y_{5}(p)+Y_{6}(p)\right] .
$$

COROLLARY 4.1. (1) A Legendre map of type (a) is a oriented hypershpere.

(2) A Legendre map of type (b) is a cyclide of Dupin.

(3) A Legendre map of type (c) (type (d)) is a canal surface if the function $y \equiv 0(x \equiv 0)$.

Proof. (1) $[K]$ is constant since $d Y_{6}=0$, so $\lambda$ is a oriented hypersphere.

(2) Let $X_{1}, X_{2}$ are the principal vectors corresponding to $\left[K_{1}\right],\left[K_{2}\right]$ respectively; i.e. $X_{1}, X_{2}$ are vectors in $T_{p} M$ such that $\phi_{1}\left(X_{1}\right)=0, \phi_{2}\left(X_{2}\right)=0$. If $\lambda$ is of type (b), then

$$
d K_{1}=2 \phi_{1} Y_{3}, \quad d K_{2}=-\phi_{2} Y_{4},
$$

and hence $d K_{1}\left(X_{1}\right)=d K_{2}\left(X_{2}\right)=0$. Thus along every line of curvature in $M$ the corresponding curvature sphere is constant; i.e. $\lambda$ is a cyclide of Dupin.

(3) Let $\lambda$ be of type (c). We change the functions $k^{1}, k^{2}$ in (2.3) so that $h^{1}=$ $4 k^{1}, h^{2}=2 k^{2}$. Then

$$
d K_{1}=2\left\{\phi_{1} Y_{3}+\left(\frac{h^{1}-2}{4} \phi_{1}+\frac{h^{2}}{2} \phi_{2}\right) Y_{5}+\left(\frac{h^{1}-2}{4} \phi_{1}+\frac{h^{2}}{2} \phi_{2}\right) Y_{6}\right\}
$$




$$
d K_{2}=2\left\{-\phi_{2} Y_{4}+\left(-\frac{h^{1}+1}{2} \phi_{1}-h^{2} \phi_{2}\right) Y_{5}+\left(\frac{h^{1}-1}{2} \phi_{1}+h^{2} \phi_{2}\right) Y_{6}\right\} .
$$

Let $\lambda$ be of type (d). We change the functions $k^{1}, k^{2}$ in (2.5) so that $h^{1}=-2 k^{1}$, $h^{2}=-4 k^{2}$. Then

$$
\begin{aligned}
& d K_{1}=2\left\{\phi_{1} Y_{3}+\left(h^{1} \phi_{1}+\frac{h^{2}-1}{2} \phi_{2}\right) Y_{5}+\left(h^{1} \phi_{1}+\frac{h^{2}+2}{2} \phi_{2}\right) Y_{6}\right\} \\
& d K_{2}=2\left\{-\phi_{2} Y_{4}+\left(-\frac{h^{1}}{2} \phi_{1}-\frac{h^{2}+2}{4} \phi_{2}\right) Y_{5}+\left(\frac{h^{1}}{2} \phi_{1}+\frac{h^{2}+2}{4} \phi_{2}\right) Y_{6}\right\} .
\end{aligned}
$$

Thus if $\lambda$ is of type (c) and $k^{2}=h^{2} \equiv 0$, then only [ $\left.K_{1}\right]$ is constant along $X_{1}$, and if $\lambda$ is of type (d) and $k^{1}=h^{1} \equiv 0$, then only $\left[K_{2}\right]$ is constant along $X_{2}$; such $\lambda$ is classically called a canal surface.

Let $\lambda$ be of type (e). We change the functions $k^{1}, k^{2}$ in (2.7) so that $h^{1}=$ $-2 k^{1}-1, h^{2}=2 k^{2}+1$. Then

$$
\begin{aligned}
& d K_{1}=2\left\{\phi_{1} Y_{3}+\left(h^{1} \phi_{1}+\frac{h^{2}-1}{2} \phi_{2}\right) Y_{5}+\left(h^{1} \phi_{1}+\frac{h^{2}+2}{2} \phi_{2}\right) Y_{6}\right\} \\
& d K_{2}=2\left\{-\phi_{2} Y_{4}+\left(-\frac{h^{1}+1}{2} \phi_{1}-h^{2} \phi_{2}\right) Y_{5}+\left(\frac{h^{1}-1}{2} \phi_{1}+h^{2} \phi_{2}\right) Y_{6}\right\} .
\end{aligned}
$$

Hence we can distinguish the type of a given Legendre map $\lambda: M_{2} \rightarrow T_{1} S^{3}$ which has two curvature spheres $\left[K_{1}\right],\left[K_{2}\right]$ with multiplicity 1 in the following way. Set

$$
\begin{aligned}
& d K_{1}=2 \phi_{1} Y_{3}+\left(A \phi_{1}+B \phi_{2}\right) K_{1}+C \phi_{2} K_{2}, \\
& d K_{2}=-2 \phi_{2} Y_{4}+D \phi_{1} K_{1}+\left(E \phi_{1}+F \phi_{2}\right) K_{2},
\end{aligned}
$$

where $A, B, C, D, E, F$ are some smooth functions of $M$. If $C=0, D=0$, then $\lambda$ is of type (b); i.e. a cyclide of Dupin. If $C=0, D \neq 0(C \neq 0, D=0)$, then $\lambda$ is of type (c) (type (d)); moreover if $B=0(E=0)$, then $\lambda$ is a canal surface. If $C \neq 0, D \neq 0$, then $\lambda$ is of type (e).

Finally we obtain the necessary and sufficient condition that two surfaces are Lie equivalent by virtue of Theorem 3.1 and the theorem in Section 2.2.

COROLlary 4.2. (a) All oriented spheres in $T_{1} S^{3}$ are Lie equivalent.

(b) All cyclides of Dupin in $T_{1} S^{3}$ are Lie equivalent.

(c) Let $\lambda: M \rightarrow T_{1} S^{2}, \tilde{\lambda}: \tilde{M} \rightarrow T_{1} S^{2}$ be smooth surfaces of type (c), (d) or (e). Let $k^{i}$, $\tilde{k}^{2}$ be the smooth functions which are defined in Theorem 2.1 with respect to $\lambda, \tilde{\lambda}$ respectively. Surfaces $\lambda$ and $\tilde{\lambda}$ are Lie equivalent if and only if there exists a one-to-one 
correspondance $\varphi: M \rightarrow \tilde{M}$ such that $k^{i}=\varphi^{*} \tilde{k}^{i}$ for all $i$.

\section{REFERENCES}

[C] T. E. Cecil, Lie sphere geometry, Springer-Verlag, New York, 1992.

[CC] T. E. Cecil and S. S. Chern, Tautness and Lie sphere geometry, Math. Ann., 278 (1987), 381-399.

[Gri] P. Griffiths, On Cartan's method of Lie groups and moving frames as applied to uniqueness and existence questions in differential geometry, Duke. Math. J., 41 (1974), 775-814.

[J] G. R. Jensen, Higher order contact of submanifolds of homogeneous spaces, Lecture Notes in Math., 610, Springer-Verlag, Berlin Heidelberg New York, 1977.

[M] R. Miyaoka, Lie contact structure and normal Cartan connections, Kodai. Math. J., 14 (1991), 13-41.

[P] U. Pinkall, Dupin hypersurfaces, Math., 270 (1985), 427-440.

[SY] H. Sato and K. Yamaguchi, Lie contact manifolds, Geometry of manifolds (K. Shiohama ed.) pp. 191-238, Academic Press, Boston, 1989.

[Y] T. Yamazaki, Dupin hypersurfaces and Lie sphere geometry, (in Japanese), Master thesis at Tôhoku University (1988).

[YY] T. Yamazaki and A. Y. Yoshikawa, Plane curves in Lie sphere geometry, Kodai Math. J., to appear.

Takayoshi Yamazaki

Sokahigashi High School

Kakinoki-cho 1110 Soka Saitama 340

Japan

Atsuko Yamada Yoshikawa

Graduate School of Polymathematics

Nagoya University

Chikusa-ku Nagoya 464-01

Japan 